\title{
A Multiattribute Decision-Making Framework: VIKOR Method with Complex Spherical Fuzzy N-Soft Sets
}

\author{
Muhammad Akram (i), ${ }^{1}$ Maria Shabir, ${ }^{1}$ Arooj Adeel ${ }^{(1)},{ }^{2}$ and Ahmad N. Al-Kenani ${ }^{3}$ \\ ${ }^{1}$ Department of Mathematics, University of the Punjab, New Campus, Lahore, Pakistan \\ ${ }^{2}$ Department of Mathematics, University of Education, Bank Road Campus, Lahore, Pakistan \\ ${ }^{3}$ Department of Mathematics, Faculty of Science, King Abdulaziz University, P.O. Box 80219, Jeddah 21589, Saudi Arabia \\ Correspondence should be addressed to Muhammad Akram; m.akram@pucit.edu.pk
}

Received 3 July 2021; Revised 4 August 2021; Accepted 12 August 2021; Published 31 August 2021

Academic Editor: Naeem Jan

Copyright ( 92021 Muhammad Akram et al. This is an open access article distributed under the Creative Commons Attribution License, which permits unrestricted use, distribution, and reproduction in any medium, provided the original work is properly cited.

\begin{abstract}
In this paper, we set forth a framework for solving a multiattribute group decision-making (MAGDM) problem, namely, the selection of a firm for participation in a Saudi oil refinery project in Pakistan. This project will prove a key success factor for the economic growth of Pakistan due to its enormous economic impact on the energy sector, industrial development, commerce, transportation, and so on. This multiplicity justifies that several intricate components comprising both intrinsic and external attributes should be adequately evaluated for the selection of such a firm, that is, the formulation of this question as a MAGDM problem. Nonbinary evaluation with two-dimensional ambiguity and uncertainty in the parameters are general concerns in modern literature, and they fit into this problem. Within this context, one of the most superior and amenable theories (complex spherical fuzzy $N$-soft sets, henceforth $C \mathcal{S} F N S_{f} S s$ ) shall be used to formulate a new comprehensive method, known as complex spherical fuzzy $N$-soft-VIKOR $\left(C \mathcal{S} F N S_{f}\right.$-VIKOR) method. According to the general spirit of the benchmark technique, the normalized Euclidean distances and the weights of the attributes are jointly handled, and as consequence, two main features ("maximum group utility" and "minimum individual regret") are acquired. The coefficient strategy with reference to group utility measure and individual regret measure of opponents are employed for the compromise measure. Armed with this novel tool, we single out the most feasible firm according to the preference order of the alternatives examined by the decision-makers on the subject of linear normalized weights of experts and attributes. Furthermore, a comparative analysis justifies the $C \mathcal{S} F$-VIKOR method, and some results prove its capabilities and validity. Moreover, a sensitivity test certifies the stability of the proposed method.
\end{abstract}

\section{Introduction}

In 1998, the VIKOR approach was drafted by Opricovic [1] as a multiattribute decision-making (MADM) method. In the setting of civil engineering, this system attempted to find a compromise solution based on two dominant principles (group utility measure and individual regret of opponent), where the compromise solution means a decision done by generic involvement. Due to the paradigm of maximum group utility and minimum individual regret, the feasible solution closest to the best values and most distant from the worst value is resolved by the recourse to the $L_{p}$-metric as an aggregation function, according to Opricovic. Opricovic and
Tzeng [2] extended the VIKOR theory for MADM for the postearthquake reconstruction problem in Central Taiwan and the selection of destination for the mountain climber, respectively. Yazdani-Chamzini et al. [3] employed the modified version of the VIKOR method along with the combination of TOPSIS, MOORA, additive weighting, and ratio assessment techniques in order to address a multicriteria decision-making (MCDM) problem relating to renewable energy resources.

People habitually live with real-life properties that are not sufficiently precise and not fully objective, such as "beautiful," "tall," or "experienced." Many decision-related problems must take into account such properties. To 
contend with such vague and uncertain data, Zadeh [4] presented the notion of fuzzy set. Suh et al. [5] assessed the mobile service quality through the proposed groundbreaking fuzzy-VIKOR method using an integrated weighted approach. Lee et al. [6] extended the VIKOR technique for the MAGDM problem of detection of flood risks under the fuzzy frame. Li and Liu [7] presented the VIKOR with the combination of the QUALIFLEX method on the basis of trapezoidal fuzzy numbers corresponded to each two-dimensional linguistic data. Chang [8] disclosed the situation about the private and governmental hospital agencies in Taiwan with the help of the new fuzzy-VIKOR approach. Wang and Chang [9] resolved the MAGDM problem through fuzzy-VIKOR methodology. Another MAGDM problem, the selection of machine tools evaluated by the fuzzy-VIKOR approach is introduced by $\mathrm{Wu}$ et al. [10].

In accordance with fuzzy descriptions, the degree of truthiness (or satisfaction), collected from the closed unit interval $[0,1]$, denotes the correspondence of an object related to a parameter. Under these interpretations, its inability to represent the nonassociation of the object related to that particular parameter is quite obvious. For this reason, in 1986, Atanassov [11] eradicated this obstacle through the extended concept of intuitionistic fuzzy set (IFS) and confronted the vague opinion through both a degree of truthiness $\phi$ and a degree of falsity $\chi$, which are jointly subject to the constraint: $\phi+\chi \leq 1$. Roostaee et al. [12] developed the theoretical background for an IF-VIKOR method to rank the suppliers following the opinions of decision-makers, and Gupta et al. [13] extended the IF-VIKOR ideology for allocating the best environment for plantation. Krishankumar et al. [14] resolved a personnel selection problem through the extended version of the IF-VIKOR method.

Pythagorean fuzzy sets $\left(P_{y} F S s\right)$ evolved as an extension of IFSs. Introduced by Yager [15], $P_{y} F S$ also deal with both degree of truth $\phi$ and degree of falsity $\chi$, but they are under the modified condition: $\phi^{2}+\chi^{2} \leq 1$. Gul et al. [16] extended the VIKOR based approach within the field of $P_{y} F S s$ and evaluated the safety risks in the mine industry. Rani et al. [17] proposed the $P_{y} F$-VIKOR method within the tool of entropy and divergence measures for the selection of renewable energy technology in India. Ma et al. [18] introduced the group decision-making framework using complex Pythagorean fuzzy information.

People habitual nature sometimes has a neutral judgment. This is generally exhibited in certain problems or events like voting situations. Therefore, along with the judgments of yes or no, there is often a need for an abstain part of the opinion (possibly related to the satisfaction of a particular parameter). Since the Pythagorean fuzzy set is not able to handle such part of the decisional attitude, Cuong [19] introduced picture fuzzy set (PFS) along with the degree of truth $\phi$, remain neutral $\psi$, and degree of falsity $\chi$ but with their range limited by an inequality: $\phi+\psi+\chi \leq 1$. Meksavang et al. [20] extended the decision-making approach based on the VIKOR methodology for the picture fuzzy environment, which was applied for sustainable suppliers along with an application in the beef industry. Liu and You
[21] presented the PF-VIKOR technique for green supplier evaluation and selection.

However, PFSs are very reliable to deal with imprecision and fuzziness and still useless when the sum of the degrees of truth, neutrality, and falsity exceeds 1 . To get over this drawback, Gundogdu and Kahraman [22-25] worked out a model with a relaxed condition, introduced the idea of spherical fuzzy sets ( $\mathcal{S} F S s$ ), and employed them on MADM problems. Later on, Mahmood et al. [26] proposed $T$-spherical fuzzy sets, as an extension of $\mathcal{S} F S$. Gundogdu et al. $[27,28]$ developed the theory for the $\delta F$-VIKOR method and applied it to the MADM problem for waste management problems and selection of site, respectively.

The aforementioned models of fuzzy knowledge have a one-dimensional structure. This pattern is incompatible with certain types of problems. Ramot et al. [29] developed a fuzzy set model in two-directional frames, known as complex fuzzy set (CFS). In this model, the degree of truth is $\phi=t e^{i 2 \pi \zeta}$, which is divided into amplitude term $t$ and periodic term $\zeta$ that belongs to the unit closed interval. For the sake of decision-making, complex fuzzy aggregation operators are defined by Akram and Bashir [30]. Alkouri and Salleh [31] generalized the ideology to a complex intuitionistic fuzzy set (CIFS), which accounts for both degree of truthiness $\phi=t e^{i 2 \pi \zeta}$ and falsity $\chi=f e^{i 2 \pi \rho}$ along with the constraints $t+f \leq 1$ and $\zeta+\rho \leq 1$. Ullah et al. [32] introduced a complex Pythagorean fuzzy set $\left(C P_{y} F S\right)$ with degrees of truth $\phi=t e^{i 2 \pi \zeta}$ and falsity $\chi=f e^{i 2 \pi \rho}$ within the complex unit circle and restricted by the constraints $t^{2}+$ $f^{2} \leq 1$ and $\zeta^{2}+\rho^{2} \leq 1$. Currently, Akram et al. [33] presented the model of a complex spherical fuzzy set $(C \mathcal{S} F S)$ representing three degrees of truth $\phi=t e^{i 2 \pi \zeta}$, neutrality $\psi=q e^{i 2 \pi \rho}$, and falsity $\chi=f e^{i 2 \pi \rho}$ subjected to conditions $t^{2}+$ $q^{2}+f^{2} \leq 1$ and $\zeta^{2}+\varrho^{2}+\rho^{2} \leq 1$. They also extended the VIKOR method under the $C \mathcal{S} F$ environment. The yielding and tractable conditions of the $C \mathcal{S} F$ representation make it a privileged framework for the modelization of two-dimensional ambiguous knowledge. In the advantageous $C \mathscr{S F}$-VIKOR technique, decision-makers scrutinize the competencies of the feasible choices with reference to the preferred criteria and indicate initial observation through the use of linguistic information, which further enhanced the virtues of complex spherical fuzzy numbers.

Though CSFSs are highly competent and skillful, they neglect the possibility of nonbinary parameterized information. In this panorama, Akram et al. [34] presented the concept of complex spherical fuzzy $N$-soft sets $\left(C \mathscr{S} F N S_{f} S s\right)$ within the TOPSIS methodology, which has the ability to look over the modern real-life ranking problems in every field of science. In relation to this, the idea of $N$-soft sets $\left(N S_{f} S s\right)$ was presented by [35] as an extension of soft set theory [36], which accommodates all kind of attributes. Soon afterwards, Akram et al. [37] explored the scope of $N$-soft sets and put forward hybrid models, namely, fuzzy $N$-soft sets $\left(F N S_{f} S s\right)$, intuitionistic fuzzy $N$-soft sets $\left(I F N S_{f} S\right)$, and hesitant $N$-soft sets. Recently, Zhang et al. [38] combined $N$-soft theory with $P_{y} F S s$ and proposed the novel model of Pythagorean fuzzy $N$-soft set $\left(P_{y} F N S_{f} S\right)$. The

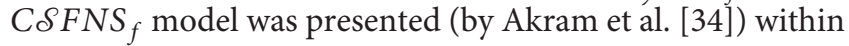


research conducive to an extension of the TOPSIS method that can deal with MAGDM problems based on $C \mathcal{S} F N S_{f}$ information. For other notations and applications, the readers are referred to [39-44].

In modern times, ranking evaluation systems (instead of linguistic information) can be in use as a primary source in furtherance of decision-making problems and surveys. The $C \mathcal{S F}$-VIKOR technique (proposed by Akram et al. [33]) is unqualified for situations comprising parameterized ranking information. Therefore, we develop a novel technique, namely, the $C \mathcal{S} F N S_{f}$-VIKOR method. The delineation and explanation related to this technique do not pertain to any of the existing techniques; hence, it requires a concrete analysis. Of course, the proposed model successfully evaluates decision-making problems related to the modern era. The purpose of our concept is to extend the VIKOR method under the circumstances of $C \mathcal{S} F N S_{f} S s$. Its motivations are given as follows:

(i) The two-dimensional influential technique of $C \mathscr{S F}$-VIKOR is inadequate to operate with data comprising ordered grades along with two or several parameters.

(ii) The fuzzy $\mathrm{N}$-soft models, along with intuitionistic fuzzy $N$-soft, Pythagorean fuzzy $N$-soft, and complex Pythagorean fuzzy $\mathrm{N}$-soft environments provide an array of models that operate under parameterized ranking systems. But they are all useless when it comes to incorporating uncertain data containing neutral opinions.

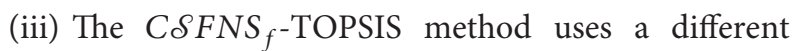
methodology but ultimately computes a feasible solution, which merely does not take into account the relative importance of distances from the ideal solution.

(iv) These limitations provoke the development of

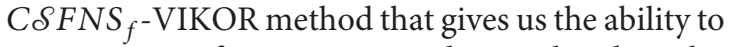
incorporate information regarding ordered grades among complex valued degrees of truth, neutrality, and falsity.

The main aim of our proposed idea is to develop the methodology of VIKOR within the hybrid model of $C \delta F N S_{f} S s$ specifically for the solution of MAGDM problems precisely. The contributions of this paper are as follows:

(i) We introduce a hybrid MAGDM VIKOR approach whose structure is based on $C \mathcal{S} F N S_{f} S s$, and it is known as $C \mathcal{S} F N S_{f}$-VIKOR technique. This methodology qualifies for situations that comprise parameterized ranking information; hence, it successfully evaluates a large proportion of existing MAGDM problems, as well as decision-making problems with nonbinary parameterized information as an initial assessment.

(ii) Linear normalized weights of experts and attributes are defined along with normalized Euclidean distance for sake of maximum group utility measure, individual regret, and compromise ranking whose values are arranged in ascending order for final decisions.

(iii) The presented technique is supported through a real-life application from the grounds of economy and business assisted by comparative analysis and sensitivity tests.

The rest of the article is structured as follows. Section 2 contains preliminaries from $C \mathcal{S} F N S_{f}$ model [34] with some operations, comparison rule, and averaging operator. In Section 3, a descriptive theory for $C \mathcal{S} F N S_{f}$-VIKOR method is developed along with a flowchart. Section 4 interpreted a real-life MAGDM problem of firm's selection for the Saudi oil refinery project. Section 5 narrated the proposed method by a sensitivity test. In Section 6, we compare our proposed method with the existing techniques. In Section 7, the merits of the $C \mathscr{S F N S} S_{f}$-VIKOR method are clarified. In the end, we present concluding remarks and mention some future research work in Section 8.

\section{Complex Spherical Fuzzy N-Soft Sets}

Definition 1 (see [34]). Let $J$ be a nonempty set and $\mathscr{X} \subseteq T$, whereas $T$ be a set of parameters (or attributes), and $C=$ $\{0,1,2, \ldots, N-1\}$ be a set of grades level with $N \in\{2,3, \ldots$,$\} . Then a complex spherical fuzzy N$-soft set $\left(\operatorname{CSFNS}_{f} S\right)$ on $\mathscr{X}$ is denoted by a triplet $\left(\ddot{E}_{H}, \mathscr{X}, N\right)$, defined as follows:

$$
\begin{aligned}
\ddot{E}_{H}\left(x_{a}\right) & =\left\{\left\langle\left(\ddot{E}\left(x_{a}\right), H\left(x_{a}\right)\right)\right\rangle: x_{a} \in X\right\} \\
& =\left\{\left\langle\left(\left(j_{b}, c_{a}^{b}\right),\left(\phi_{b a}, \psi_{b a}, \chi_{b a}\right)\right)\right\rangle\right\} \\
& =\left\{\left\langle\left(\left(j_{b}, c_{a}^{b}\right),\left(t_{w s} e^{i 2 \pi \zeta_{w s}}, q_{b a} e^{i 2 \pi e_{b a}}, f_{b a} e^{i 2 \pi \rho_{b a}}\right)\right)\right\rangle\right\},
\end{aligned}
$$

along with the assumption of $\ddot{E}: \mathscr{X} \longrightarrow 2^{J \times H}$, where $N S_{f} S$ be defined on $J$, and $H$ is a function from $\mathscr{X}$ to $\mathbb{C} \mathcal{S} \mathbb{F N}$. The notation $\mathbb{C} \mathcal{S} \mathbb{F}$ denotes the collection of all complex spherical fuzzy numbers of $J$, and $t_{b a}, \zeta_{b a}, q_{b a}, \varrho_{b a}, f_{b a}$, and $\rho_{b a}$ will be taken from unit closed interval, with constraint

$$
\begin{aligned}
& 0 \leq t_{b a}^{2}+q_{b a}^{2}+f_{b a}^{2} \leq 1, \\
& 0 \leq \zeta_{b a}^{2}+\varrho_{b a}^{2}+\rho_{b a}^{2} \leq 1 .
\end{aligned}
$$

The term $c_{a}^{b}$ denotes the ranking of attributes for the alternative $j_{b}$, for all $j_{b} \in J$.

Definition 2 (see [34]). Let $\ddot{E}_{H}\left(x_{a}\right)=\left(\left(j_{b}, c_{a}^{b}\right)\right.$, $\left.t_{b a} e^{i 2 \pi \zeta_{b a}}, q_{b a} e^{i 2 \pi \mathrm{\rho}_{b a}} f_{b a} e^{i 2 \pi \rho_{b a}}\right)$ be a $C \mathcal{S} F N S_{f} S$. Then the complex spherical fuzzy $N$-soft number $\left(C \mathscr{S} F N S_{f} N\right)$ is defined as follows:

$$
\mathrm{F}_{b a}=\left(c_{a}^{b}, t_{b a} e^{i 2 \pi \zeta_{b a}}, q_{b a} e^{i 2 \pi e_{b a}}, f_{b a} e^{i 2 \pi \rho_{b a}}\right),
$$

and the hesitancy degree is defined as follows: 


$$
\Omega_{\mathrm{Fba}}=\sqrt{1-\left(t_{b a}^{2}+q_{b a}^{2}+f_{b a}^{2}\right)} e^{i 2 \pi \sqrt{1-\left(\zeta_{b a}^{2}+e_{b a}^{2}+\rho_{b a}^{2}\right)}} .
$$

Definition 3. (see [34]). Let $\mathrm{F}_{b a}=\left(c_{a}^{b}, t_{b a} e^{i 2 \pi \zeta_{b a}}\right.$, $\left.q_{b a} e^{i 2 \pi \rho_{b a}}, f_{b a} e^{i 2 \pi \rho_{b a}}\right)$ be $\operatorname{CSFNS}_{f} N$. The score function $\mathrm{Sc}\left(\mathrm{F}_{b a}\right)$ is defined as follows:

$$
S c_{\mathrm{Fba}}=\left(\frac{c_{a}^{b}}{N-1}\right)^{2}+\left(t_{b a}^{2}-q_{b a}^{2}-f_{b a}^{2}\right)+\left[\zeta_{b a}^{2}-\varrho_{b a}^{2}-\rho_{b a}^{2}\right],
$$

where $S c_{F_{b a}} \in[-2,3]$. The accuracy function $A c\left(\mathrm{~F}_{b a}\right)$ is defined as follows:

$$
A c_{\mathrm{F} b a}=\left(\frac{c_{a}^{b}}{N-1}\right)^{2}+\left(t_{b a}^{2}+q_{b a}^{2}+f_{b a}^{2}\right)+\left[\zeta_{b a}^{2}+\varrho_{b a}^{2}+\rho_{b a}^{2}\right],
$$

Definition 4 (see [34]). Let $\mathrm{F}_{b l}=\left(c_{l}^{b}, t_{l j} e^{i 2 \pi \zeta_{b l}}, q_{b b} e^{i 2 \pi \mathrm{e}_{b l}}\right.$, $\left.f_{b l} e^{i 2 \pi \rho_{b l}}\right)$ and $\mathrm{F}_{b a}=\left(c_{a}^{b}, t_{b a} e^{i 2 \pi \zeta_{b a}}, q_{b a} e^{i 2 \pi \mathrm{e}_{b a}}, f_{b a} e^{i 2 \pi \rho_{b a}}\right)$ be two $\mathrm{CSFNS}_{f} \mathrm{Ns}$.

(1) If $S c_{\mathrm{Fbl}}<S c_{\mathrm{Fba}}$, then $\mathrm{Fbl}_{b l}<\mathrm{F}_{b a}\left(\mathrm{~F}_{b l}\right.$ is inferior to $\left.\mathrm{F}_{b a}\right)$

(2)If $S c_{\mathrm{Fbl}_{b}}>S c_{\mathrm{Fba}}$, then $\mathrm{Fbl}_{b l}>\mathrm{Fba}_{b a}$ ( $\mathrm{Fbl}$ is superior to $\mathrm{F}_{b a}$ )

(3) If $S c_{F b l}=S c_{F b a}$, then

(i) $A c_{\mathrm{F}_{b l}}<A c_{\mathrm{F}_{b a}}$, then $\mathrm{F}_{b l}<\mathrm{F}_{b a}\left(\mathrm{~F}_{b l}\right.$ is inferior to $\left.\mathrm{F}_{b a}\right)$

(ii) $A c_{\mathrm{F}_{b b}}>A c_{\mathrm{F}_{b a}}$, then $\mathrm{F}_{b l}>\mathrm{F}_{b a}\left(\mathrm{~F}_{b l}\right.$ is superior to $\left.\mathrm{F}_{b a}\right)$

(iii) $A c_{F_{b l}}=A c_{F_{b b}}$, then $\mathrm{F}_{b l} \sim \mathrm{F}_{b a}$ ( $\mathrm{F}_{b l}$ is equivalent to $\mathrm{Fba})$

Definition 5 (see [34]). Let $\mathrm{F}_{b l}=\left(c_{l}^{b}, t_{b l} e^{i 2 \pi \zeta_{b l}}, q_{b l} e^{i 2 \pi e_{b l}}\right.$, $\left.f_{b l} e^{i 2 \pi \rho_{b l}}\right)$ and $\mathrm{F}_{b a}=\left(c_{a}^{b}, t_{b a} e^{i 2 \pi \zeta_{b a}}, q_{b a} e^{i 2 \pi e_{b a}}, f_{b a} e^{i 2 \pi \rho_{b a}}\right)$ be two $\mathrm{CSFNS}_{f} N s$ and $\lambda>0$. Some operations for CSFNS ${ }_{f} N s$ are as follows:

where $A c_{\mathrm{Fba}} \in[0,3]$.

$$
\begin{aligned}
\lambda_{\mathrm{F} b l} & =\left(c_{l}^{b}, \sqrt{\left[1-\left(1-t_{b l}^{2}\right)^{\lambda}\right]} e^{i 2 \pi} \sqrt{\left[1-\left(1-\zeta_{b l}^{2}\right)^{\lambda}\right]}, q_{b l}^{\lambda} e^{i 2 \pi \varrho_{b l}^{\lambda}}, f_{b l}^{\lambda} e^{i 2 \pi \rho_{b l}^{\lambda}}\right), \\
\mathrm{F}_{b l}^{\lambda} & =\left(c_{l}^{b}, t_{b l}^{\lambda} e^{i 2 \pi \zeta_{b l}^{\lambda}}, \sqrt{\left[1-\left(1-v_{b l}^{2}\right)^{\lambda}\right]} e^{i 2 \pi} \sqrt{\left[1-\left(1-\varrho_{b l}^{2}\right)^{\lambda}\right]}, \sqrt{\left[1-\left(1-f_{b l}^{2}\right)^{\lambda}\right]} e^{i 2 \pi \sqrt{\left[1-\left(1-\rho_{b l}^{2}\right)^{\lambda}\right]}}\right), \\
\mathrm{F}_{b l} \oplus \mathrm{F}_{b a} & =\left(\max \left(c_{l}^{b}, c_{a}^{b}\right), \sqrt{t_{b l}^{2}+t_{b a}^{2}-t_{b l}^{2} t_{b a}^{2}} e^{i 2 \pi \sqrt{\zeta_{\zeta l}^{2}+\zeta_{b a}^{2}-\zeta_{b l}^{2} l_{b a}^{2}}}, q_{b l} q_{b a} e^{i 2 \pi \varrho_{b l} l_{b a}}, f_{b l} f_{b a} e^{i 2 \pi \rho_{b l} \rho_{b a}}\right), \\
\mathrm{F}_{b l} \otimes \mathrm{F}_{b a} & =\left(\min \left(c_{l}^{b}, c_{a}^{b}\right), t_{b l} t_{b a} e^{i 2 \pi \zeta_{b l} \zeta_{b a}}, \sqrt{v_{l j}^{2}+v_{k j}^{2}-v_{l j}^{2} v_{k j}^{2}} e^{i 2 \pi \sqrt{\varrho_{b l}^{2}+\varrho_{b a}^{2}-\varrho_{b l}^{2} \varrho_{b a}^{2}}}, \sqrt{f_{l j}^{2}+f_{k j}^{2}-f_{l j}^{2} f_{k j}^{2}} e^{i 2 \pi \sqrt{\rho_{b l}^{2}+\rho_{b a}^{2}-\rho_{b l}^{2} \rho_{b a}^{2}}}\right) .
\end{aligned}
$$

Proposition 1 (see [34]). Let $\mathrm{F}_{b a}=\left(c_{a}^{b}, t_{b a} e^{i 2 \pi \zeta_{b a}}\right.$, $\left.q_{b b} e^{i 2 \pi \mathrm{e}_{b a}}, f_{b a} a^{i 2 \pi \rho_{b a}}\right)$ and $\mathrm{F}_{b l}=\left(c_{l}^{b}, t_{b l} e^{i 2 \pi \zeta_{b l}}, q_{b l} l^{i 2 \pi \mathrm{e}_{b l}}, f_{b l} e^{i 2 \pi \rho_{b l}}\right)$ be two $C \mathcal{S} F N S_{f} N s$ and $\lambda>0$, then the following properties hold:

(1) $\mathrm{F}_{b a} \oplus \mathrm{F}_{b l}=\mathrm{F}_{b l} \oplus \mathrm{F}_{b a}$

(2) $\mathrm{F}_{b a} \otimes \mathrm{F}_{b l}=\mathrm{F}_{b l} \otimes \mathrm{F}_{b a}$

(3) $\lambda_{\mathrm{F}_{b a}} \oplus \lambda_{\mathrm{F}_{b l}}=\lambda\left(\mathrm{F}_{b l} \oplus \mathrm{F}_{b a}\right), \lambda>0$
(4) $\lambda_{1} \mathrm{~F}_{b a} \oplus \lambda_{2} \mathrm{~F}_{b a}=\left(\lambda_{1}+\lambda_{2}\right)_{b a}, \lambda_{1}, \lambda_{2}>0$

(5) $\mathrm{F}_{b a}^{\lambda} \otimes \mathrm{F}_{b l}^{\lambda}=\left(\mathrm{F}_{b l} \otimes \mathrm{F}_{b a}\right)^{\lambda}, \lambda>0$

(6) $\mathrm{F}_{b a}^{\lambda_{1}} \otimes \mathrm{F}_{b a}^{\lambda_{2}}=\mathrm{F}_{b a}^{\left(\lambda_{1}+\lambda_{2}\right)} \cdot \lambda_{1}, \lambda_{2}>0$

Proof

(1) $\mathrm{F}_{b a} \oplus \mathrm{F}_{b l}$

$$
\begin{aligned}
& =\left(\max \left(c_{a}^{b}, c_{l}^{b}\right),\left[\sqrt{t_{b a}^{2}+t_{b l}^{2}-t_{b a}^{2} t_{b l}^{2}}\right] e^{i 2 \pi\left[\sqrt{\zeta_{b a}^{2}+\zeta_{b l}^{2}-\zeta_{b a}^{2} \zeta_{b l}^{2}}\right]},\left[q_{b a} q_{b l}\right] e^{i 2 \pi\left[\varrho_{b a} \varrho_{b l}\right]},\left[f_{b a} f_{b l}\right] e^{i 2 \pi\left[\rho_{b a} \rho_{b l}\right]}\right), \\
& =\left(\max \left(c_{l}^{b}, c_{a}^{b}\right),\left[\sqrt{t_{b l}^{2}+t_{b a}^{2}-t_{b l}^{2} t_{b a}^{2}}\right] e^{i 2 \pi\left[\sqrt{\zeta_{b l}^{2}+\zeta_{b a}^{2}-\zeta_{b l}^{2} \zeta_{b a}^{2}}\right]},\left[q_{b l} q_{b a}\right] e^{i 2 \pi\left[\rho_{b l} \varrho_{b a}\right]},\left[f_{b l} f_{b a}\right] e^{i 2 \pi\left[\rho_{b l} \rho_{b a}\right]}\right), \\
& =\mathrm{F}_{b l} \oplus \mathrm{F}_{b a} .
\end{aligned}
$$


(2) $\mathrm{F}_{b a} \otimes \mathrm{F}_{b l}$

$\left.=\left(\min \left(c_{a}^{b}, c_{l}^{b}\right),\left[t_{b a} t_{b l}\right] e^{i 2 \pi\left[\zeta_{b a} \zeta_{b l}\right]},\left[\sqrt{q_{b a}^{2}+q_{b l}^{2}-q_{b a}^{2} q_{b l}^{2}}\right] e^{i 2 \pi\left[\sqrt{\varrho_{b a}^{2}+\varrho_{b l}^{2}-\varrho_{b a}^{2} \varrho_{b l}^{2}}\right]},\left[\sqrt{f_{b a}^{2}+f_{b l}^{2}-f_{b a}^{2} f_{b l}^{2}}\right] e^{i 2 \pi\left[\sqrt{\rho_{b a}^{2}+\rho_{b l}^{2}-\rho_{b a}^{2} \rho_{b l}^{2}}\right.}\right]\right)$

$=\left(\min \left(c_{l}^{b}, c_{a}^{b}\right),\left[t_{b l} t_{b a}\right] e^{i 2 \pi\left[\zeta_{b l} \zeta_{b a}\right]},\left[\sqrt{q_{b l}^{2}+q_{b a}^{2}-q_{b l}^{2} q_{b a}^{2}}\right] e^{i 2 \pi\left[\sqrt{\varrho_{b l}^{2}+\varrho_{b a}^{2}-\varrho_{b l}^{2} \varrho_{b a}^{2}}\right]},\left[\sqrt{f_{b l}^{2}+f_{b a}^{2}-f_{b l}^{2} f_{b a}^{2}}\right] e^{i 2 \pi}\left[\sqrt{\rho_{b l}^{2}+\rho_{b a}^{2}-\rho_{b l}^{2} \rho_{b a}^{2}}\right]\right)$

$=\mathrm{F}_{b l} \otimes \mathrm{F}_{b a}$.

(3) $\lambda_{\mathrm{F}_{b a}} \oplus \lambda_{\mathrm{F}_{b l}}$

$=\left(c_{a}^{b},\left[\sqrt{\left[1-\left(1-t_{b a}^{2}\right)^{\lambda}\right]}\right] e^{i 2 \pi\left[\sqrt{\left[1-\left(1-\zeta_{b a}^{2}\right)^{\lambda}\right]}\right]},\left[q_{b a}^{\lambda}\right] e^{i 2 \pi\left[\varrho_{b a}^{\lambda}\right]},\left[f_{b a}^{\lambda}\right] e^{i 2 \pi\left[\rho_{b a}^{\lambda}\right]}\right)$

$\oplus\left(c_{l}^{b},\left[\sqrt{\left[1-\left(1-t_{b l}^{2}\right)^{\lambda}\right]}\right] e^{i 2 \pi\left[\sqrt{\left[1-\left(1-\zeta_{b l}^{2}\right)^{\lambda}\right]}\right]},\left[q_{b l}^{\lambda}\right] e^{i 2 \pi\left[\varrho_{b l}^{\lambda}\right]},\left[f_{b l}^{\lambda}\right] e^{i 2 \pi\left[\rho_{b l}^{\lambda}\right]}\right)$

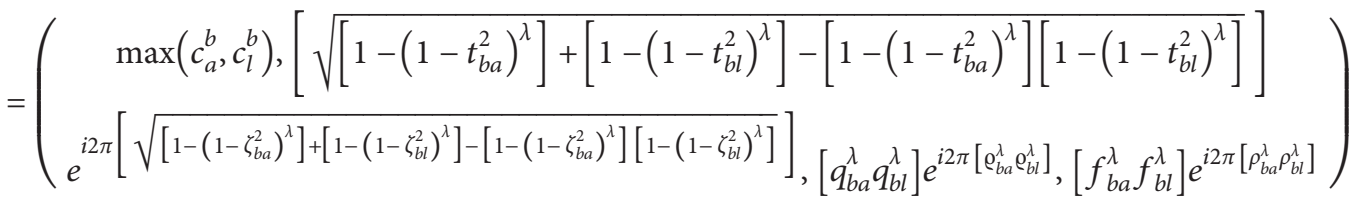

$=\left(\max \left(c_{a}^{b}, c_{l}^{b}\right),\left[\sqrt{\left[1-\left(1-t_{b a}^{2}+t_{b l}^{2}-t_{b a}^{2} t_{b l}^{2}\right)^{\lambda}\right]}\right] e^{i 2 \pi\left[\sqrt{\left[1-\left(1-\zeta_{b a}^{2}+\zeta_{b l}^{2}-\zeta_{b a}^{2} \zeta_{b l}^{2}\right)^{\lambda}\right]}\right]},\left(q_{b a} q_{b l}\right)^{\lambda} e^{i 2 \pi\left(\varrho_{b a} \varrho_{b l}\right)^{\lambda}},\left(f_{b a} f_{b l}\right)^{\lambda} e^{i 2 \pi\left(\rho_{b a} \rho_{b l}\right)^{\lambda}}\right)$

$=\lambda\left(\max \left(c_{a}^{b}, c_{l}^{b}\right),\left[\sqrt{t_{b a}^{2}+t_{b l}^{2}-t_{b a}^{2} t_{b l}^{2}}\right] e^{i 2 \pi\left[\sqrt{\zeta_{b a}^{2}+{ }_{b l}^{2}-\zeta_{b a}^{2} \zeta_{b l}^{2}}\right]},\left(q_{b a} q_{b l}\right) e^{2 \pi\left(\varrho_{b a} \varrho_{b l}\right)},\left(f_{b a} f_{b l}\right) e^{2 \pi\left(\rho_{b a} \rho_{b l}\right)}\right)$

$=\lambda\left(\mathrm{F}_{b a} \oplus \mathrm{F}_{b l}\right)$.

(4) $\lambda_{1} \mathrm{~F}_{b a} \oplus \lambda_{2} \mathrm{~F}_{b a}$

$$
\begin{aligned}
& =\left(c_{a}^{b},\left[\sqrt{\left[1-\left(1-t_{b a}^{2}\right)^{\lambda_{1}}\right]}\right] e^{i 2 \pi\left[\sqrt{\left[1-\left(1-\zeta_{b a}^{2}\right)^{\lambda_{1}}\right]}\right]},\left[q_{b a}^{\lambda_{1}}\right] e^{i 2 \pi\left[\varrho_{b a}^{\lambda_{1}}\right]},\left[f_{b a}^{\lambda_{1}}\right] e^{i 2 \pi\left[\rho_{b a}^{\lambda_{1}}\right]}\right) \\
& \oplus\left(c_{l}^{b},\left[\sqrt{\left[1-\left(1-t_{b a}^{2}\right)^{\lambda_{2}}\right]}\right] e^{i 2 \pi\left[\sqrt{\left[1-\left(1-\zeta_{b a}^{2}\right)^{\lambda_{2}}\right]}\right]},\left[q_{b a}^{\lambda_{2}}\right] e^{i 2 \pi\left[\varrho_{b a}^{\lambda_{2}}\right]},\left[f_{b a}^{\lambda_{2}}\right] e^{i 2 \pi\left[\rho_{b a}^{\lambda_{2}}\right]}\right) \\
& =\left(\begin{array}{c}
\max \left(c_{a}^{b}, c_{l}^{b}\right),\left[\sqrt{\left[1-\left(1-t_{b a}^{2}\right)^{\lambda_{1}}\right]+\left[1-\left(1-t_{b a}^{2}\right)^{\lambda_{2}}\right]-\left[1-\left(1-t_{b a}^{2}\right)^{\lambda_{1}}\right]\left[1-\left(1-t_{b a}^{2}\right)^{\lambda_{2}}\right]}\right] \\
e^{i 2 \pi\left[\sqrt{\left[1-\left(1-\zeta_{b a}^{2}\right)^{\lambda_{1}}\right]+\left[1-\left(1-\zeta_{b a}^{2}\right)^{\lambda_{2}}\right]-\left[1-\left(1-\zeta_{b a}^{2}\right)^{\lambda}\right]\left[1-\left(1-\zeta_{b a}^{2}\right)^{\lambda_{2}}\right]}\right]},\left[q_{b a}^{\lambda_{1}} q_{b a}^{\lambda_{2}}\right] e^{i 2 \pi\left[\varrho_{b a}^{\lambda_{1}} e_{b a}^{\lambda_{2}}\right]},\left[f_{b a}^{\lambda_{1}} f_{b a}^{\lambda_{2}}\right] e^{i 2 \pi\left[\rho_{b a}^{\lambda_{1}} \rho_{b a}^{\lambda_{2}}\right]}
\end{array}\right) \\
& =\left(\max \left(c_{a}^{b}, c_{a}^{b}\right),\left[\sqrt{\left[1-\left(1-t_{b a}^{2}\right)^{\left(\lambda_{1}+\lambda_{2}\right)}\right]}\right] e^{i 2 \pi\left[\sqrt{\left[1-\left(1-\zeta_{b a}^{2}\right)^{\left(\lambda_{1}+\lambda_{2}\right)}\right]}\right]},\left(q_{b a}\right)^{\left(\lambda_{1}+\lambda_{2}\right)} e^{i 2 \pi\left(\varrho_{b a}\right)^{\left(\lambda_{1}+\lambda_{2}\right)}},\left(f_{b a}\right)^{\left(\lambda_{1}+\lambda_{2}\right)} e^{\left.i 2 \pi\left(\rho_{b a}\right)^{\left(\lambda_{1}+\lambda_{2}\right)}\right)}\right) \\
& =\left(\lambda_{1}+\lambda_{2}\right)\left(c_{a}^{b}, t_{b a} e^{i 2 \pi \zeta_{b a}},\left(q_{b a}\right) e^{2 \pi\left(\varrho_{b a}\right)},\left(f_{b a}\right) e^{2 \pi\left(\rho_{b a}\right)}\right) \\
& =\left(\lambda_{1}+\lambda_{2}\right) \mathrm{F}_{b a} \text {. }
\end{aligned}
$$


Similarly, we can clarify (5) and (6).

Definition 6 (see [34]). Let $\mathrm{F}_{b a}=\left(c_{a}^{b}, t_{b a} e^{i 2 \pi \zeta_{b a}}, q_{b a} e^{i 2 \pi \varrho_{b a}}\right.$, $\left.f_{b a} e^{i 2 \pi \rho_{b a}}\right)(a=1,2, \ldots, v)$ be a collection of $C \mathcal{S} F N S_{f} N s$, then complex spherical fuzzy $N$-soft weighted average $\left(C \mathcal{S F N S} S_{f} W\right.$ ) operator is defined as follows:

$$
\begin{aligned}
& \operatorname{CSFNS}_{f} W A\left(\mathrm{~F}_{b 1}, \mathrm{~F}_{b 2}, \ldots, \mathrm{F}_{b v}\right)=\left(\bigoplus_{a=1}^{v} \lambda_{a} \mathrm{~F}_{b a}\right) \\
& =\left(\begin{array}{c}
\max _{a=1}^{v}\left(c_{a}^{b}\right), \sqrt{\left[1-\prod_{a=1}^{v}\left(1-t_{b a}^{2}\right)^{\lambda_{a}}\right]} e^{i 2 \pi \sqrt{\left[1-\prod_{a=1}^{v}\left(1-\zeta_{b a}^{2}\right)^{\lambda_{a}}\right]}}, \\
{\left[\prod_{a=1}^{v}\left(q_{b a}\right)^{\lambda_{a}}\right] e^{i 2 \pi\left[\prod_{a=1}^{v}\left(\varrho_{b a}\right)^{\lambda_{a}}\right]},\left[\prod_{a=1}^{v}\left(f_{b a}\right)^{\lambda_{a}}\right] e^{i 2 \pi\left[\prod_{a=1}^{v}\left(\rho_{b a}\right)^{\lambda_{a}}\right]}}
\end{array}\right),
\end{aligned}
$$

where $\lambda_{a}=\left(\lambda_{1}, \lambda_{1}, \ldots, \lambda_{v}\right)^{T}$ is a weighted vector of $\mathrm{F}_{b a}$ with the property that $\lambda_{a}>0$ and $\sum_{a=1}^{v} \lambda_{a}=1$, for all $(b=1,2, \ldots, m)$.

$$
\begin{aligned}
& \operatorname{CSFNS}_{f} W A\left(\mathrm{~F}_{b 1}, \mathrm{~F}_{b 2}, \ldots, \mathrm{F}_{b v}\right)=\left(\bigoplus_{a=1}^{v} \lambda_{a} \mathrm{~F}_{b a}\right) \\
& =\left(\begin{array}{c}
\max _{a=1}^{v}\left(c_{a}^{b}\right), \sqrt{\left[1-\prod_{a=1}^{v}\left(1-t_{b a}^{2}\right)^{\lambda_{a}}\right]} e^{i 2 \pi \sqrt{\left[1-\prod_{a=1}^{v}\left(1-\zeta_{b a}^{2}\right)^{\lambda_{a}}\right]}}, \\
{\left[\prod_{a=1}^{v}\left(q_{b a}\right)^{\lambda_{a}}\right] e^{i 2 \pi\left[\prod_{a=1}^{v}\left(\varrho_{b a}\right)^{\lambda_{a}}\right]},\left[\prod_{a=1}^{v}\left(f_{b a}\right)^{\lambda_{a}}\right] e^{i 2 \pi\left[\prod_{a=1}^{v}\left(\rho_{b a}\right)^{\lambda_{a}}\right]}}
\end{array}\right) . \\
& =\left(\left(c_{1}^{b}\right), \sqrt{\left[1-\left(1-t_{b 1}^{2}\right)\right]} e^{i 2 \pi \sqrt{\left[1-\left(1-\zeta_{b 1}^{2}\right)\right]}},\left[\left(q_{b 1}\right)\right] e^{i 2 \pi\left[\left(\varrho_{b a}\right)\right]},\left[\left(f_{b a}\right)\right] e^{i 2 \pi\left[\left(\rho_{b a}\right)\right]}\right) \\
& =\left(c_{a}^{b}, t_{b a} e^{i 2 \pi \zeta_{b a}}, q_{b a} e^{i 2 \pi \varrho_{b a}}, f_{b a} e^{i 2 \pi \rho_{b a}}\right) \text {. }
\end{aligned}
$$$$
\operatorname{CSFNS}_{f} W A\left(\mathrm{~F}_{b 1}, \mathrm{~F}_{b 2}, \ldots, \mathrm{F}_{b v}\right)=\lambda_{1} \mathrm{~F}_{b 1}
$$$$
=\mathrm{F}_{b 1} \text {, }
$$
following equation:
Theorem 1. Let $\mathrm{F}_{b a}=\left(c_{a}^{b}, t_{b a} e^{i 2 \pi \zeta_{b a}}, q_{b a} e^{i 2 \pi \varrho_{b a}}, f_{b a} e^{i 2 \pi \rho_{b a}}\right)$ $(a=1,2, \ldots, v)$ be $a$ collection of $\operatorname{CSFNS}_{f} N s$ and $\lambda=\left(\lambda_{1}, \lambda_{2}, \ldots, \lambda_{v}\right)^{T}$ be the weight vector then the aggregated value by applying the $C \mathcal{S} F N S_{f} W A$ operator is also a $\mathrm{CSFNS}_{f} \mathrm{~N}$ formulated as follows:
Proof. The proof is followed by mathematical induction.
The result is true for $a=1$, as equation (15) is a CSFNS S. .
Case 2. Suppose that the result hold for $a=n, n$ is a natural number, and therefore, equation (13) becomes

$$
\begin{aligned}
& \operatorname{CSFNS}_{f} W A\left(\mathrm{~F}_{b 1}, \mathrm{~F}_{b 2}, \ldots, \mathrm{F}_{b n}\right)=\left(\bigoplus_{a=1}^{n} \lambda_{a} \mathrm{~F}_{b a}\right) \\
& =\left(\begin{array}{c}
\max _{a=1}^{n}\left(c_{a}^{b}\right), \sqrt{\left[1-\prod_{a=1}^{n}\left(1-t_{b a}^{2}\right)^{\lambda_{a}}\right]} e^{i 2 \pi} \sqrt{\left[1-\prod_{a=1}^{n}\left(1-\zeta_{b a}^{2}\right)^{\lambda_{a}}\right]}, \\
{\left[\prod_{a=1}^{n}\left(q_{b a}\right)^{\lambda_{a}}\right] e^{i 2 \pi\left[\prod_{a=1}^{n}\left(\varrho_{b a}\right)^{\lambda_{a}}\right]},\left[\prod_{a=1}^{n}\left(f_{b a}\right)^{\lambda_{a}}\right] e^{i 2 \pi\left[\prod_{a=1}^{n}\left(\rho_{b a}\right)^{\lambda_{a}}\right]}}
\end{array}\right) .
\end{aligned}
$$


Now we prove for $a=n+1$. Consider

$$
\begin{aligned}
& \operatorname{CSFNS}_{f} W A\left(\mathrm{~F}_{b 1}, \mathrm{~F}_{b 2}, \ldots, \mathrm{F}_{b(n+1)}\right)=\left(\bigoplus_{a=1}^{n+1} \lambda_{a} \mathrm{~F}_{b a}\right) \\
& =\left(\begin{array}{c}
\max _{a=1}^{n}\left(c_{a}^{b}\right), \sqrt{\left[1-\prod_{a=1}^{n}\left(1-t_{b a}^{2}\right)^{\lambda_{a}}\right]} e^{i 2 \pi \sqrt{\left[1-\prod_{a=1}^{n}\left(1-\zeta_{b a}^{2}\right)^{\lambda_{a}}\right]}}, \\
{\left[\prod_{a=1}^{n}\left(q_{b a}\right)^{\lambda_{a}}\right] e^{i 2 \pi\left[\prod_{a=1}^{n}\left(\varrho_{b a}\right)^{\lambda_{a}}\right]},\left[\prod_{a=1}^{n}\left(f_{b a}\right)^{\lambda_{a}}\right] e^{i 2 \pi\left[\prod_{a=1}^{n}\left(\rho_{b a}\right)^{\lambda_{a}}\right]}}
\end{array}\right) \\
& \oplus\left(c_{n}^{b}+1, \sqrt{\left[1-\left(1-t_{b(n+1)}^{2}\right)\right]} e^{i 2 \pi \sqrt{\left[1-\left(1-\zeta_{b(n+1)}^{2}\right)\right]}},\left[\left(q_{b(n+1)}\right)\right] e^{i 2 \pi\left[\left(\varrho_{b(n+1)}\right)\right]},\right. \\
& \left.\left[\left(f_{b(n+1)}\right)\right] e^{i 2 \pi\left[\left(\rho_{b(n+1)}\right)\right]}\right)
\end{aligned}
$$

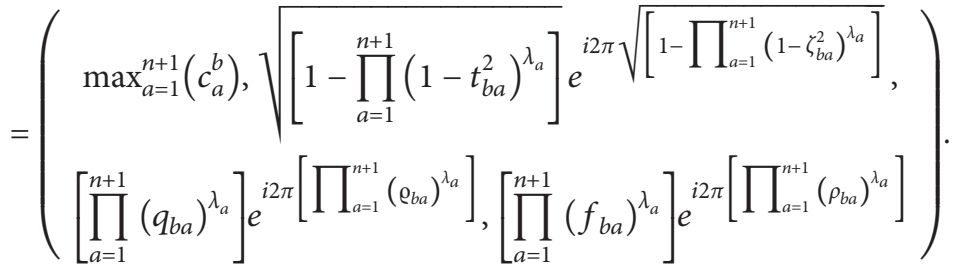

Result holds for $n+1$; hence, it is held for all natural numbers.

Theorem 2 (idempotency property). Let $\mathrm{F}_{b a}=\left(c_{a}^{b}\right.$, $\left.t_{b a} e^{i 2 \pi \zeta_{b a}}, q_{b a} e^{i 2 \pi \varrho_{b a}}, f_{b a} e^{i 2 \pi \rho_{b a}}\right)(a=1,2, \ldots, v)$ be a collection of CSFNS $\operatorname{SSs}_{f}$ and $\lambda=\left(\lambda_{1}, \lambda_{2}, \ldots, \lambda_{v}\right)^{T}$ be the weight vector of $\mathrm{F}_{b a}$, if $\mathrm{F}_{b a}=\mathrm{F}_{11}$ for all $b, a$; then

$$
\begin{aligned}
\operatorname{CSFNS}_{f} W A\left(\mathrm{~F}_{b 1}, \mathrm{~F}_{b 2}, \ldots, \mathrm{F}_{b v}\right) & =\bigoplus_{a=1}^{v}\left(\lambda_{a} \mathrm{~F}_{b a}\right) \\
& =\left(\mathrm{F}_{11}\right) .
\end{aligned}
$$

Theorem 3 (boundedness property). Let $\mathrm{F}_{b a}=\left(c_{a}^{b}\right.$, $\left.t_{b a} e^{i 2 \pi \zeta_{b a}}, q_{b a} e^{i 2 \pi \varrho_{b a}}, f_{b a} e^{i 2 \pi \rho_{b a}}\right)$ be a collection of CSFNS ${ }_{f} N s$ and for all $b, a$; then

$$
\mathrm{F}^{-} \leq C \mathcal{S F N S} S_{f} W \leq^{+}
$$

where, $\quad \mathrm{F}^{+}=\left(\max _{a} c_{a}^{b}, \max _{a}\left[t_{b a}\right] e^{i 2 \pi \max _{a}\left[\zeta_{b a}\right]}, \min _{a}\left[q_{b a}\right]\right.$ $\left.e^{i 2 \pi \min _{a}\left[\varrho_{b a}\right]}, \min _{a}\left[f_{b a}\right] e^{i 2 \pi \min _{a}\left[\rho_{b a}\right]}\right)$ and $\mathrm{F}^{-}=\left(\min _{a} c_{a}^{b}, \min _{a}\right.$ $\left[t_{b a}\right] e^{i 2 \pi \min _{a}\left[\zeta_{b a}\right]}, \quad \max _{a}\left[q_{b a}\right] e^{i 2 \pi \max _{a}\left[\varrho_{b a}\right]}, \quad \max _{a}\left[f_{b a}\right]$ $\left.e^{i 2 \pi \max _{a}\left[\rho_{b a}\right]}\right)$.

Theorem 4 (monotonicity property). Consider $\mathrm{F}_{b a}=\left(c_{a}^{b}\right.$, $\left.t_{b a} e^{i 2 \pi \zeta_{b a}}, q_{b a} e^{i 2 \pi \varrho_{b a}}, f_{b a} e^{i 2 \pi \rho_{b a}}\right) \quad$ and $\overline{\mathrm{F}}_{b a}=\left(\bar{c}_{a}^{b}, \bar{t}_{b a} e^{i 2 \pi \bar{\zeta}_{b a}}\right.$, $\left.\bar{q}_{b a} e^{i 2 \pi \bar{\varrho}_{b a}}, \bar{f}_{b a} e^{i 2 \pi \bar{\rho}_{b a}}\right)$ are two collections of CSFNS ${ }_{f} N$ s along with $c_{a}^{b} \leq \bar{c}_{a}^{b}, t_{b a} \leq \bar{t}_{b a}, \zeta_{b a} \leq \bar{\zeta}_{b a}, q_{b a} \geq \bar{q}_{b a}, \varrho_{b a} \geq \bar{\varrho}_{b a}, f_{b a} \geq \bar{f}_{b a}$, and $\rho_{b a} \geq \bar{\rho}_{b a}$; then

$$
\operatorname{CSFNS}_{f} W A\left(\mathrm{~F}_{b 1}, \mathrm{~F}_{b 2}, \ldots, \mathrm{F}_{b v}\right) \leq \operatorname{CSFNS}_{f} W A\left(\overline{\mathrm{F}}_{b 1}, \overline{\mathrm{F}}_{b 2}, \ldots, \overline{\mathrm{F}}_{b v}\right)
$$

\section{Complex Spherical Fuzzy N-Soft- VIKOR Method}

This section delineates the VIKOR method to resolve the MAGDM problems employing the domain of $C \mathcal{S} F N S_{f} S s$, which is appropriate to deal with two-dimensional data. The proposed method works out to compromise a solution in which an agreement is accomplished by mutual conductance that possesses maximum group utility and minimum individual regret.

Let $J=\left\{J_{1}, J_{2}, J_{3}, \ldots, J_{m}\right\}$ be the set of $m$ possible choices whose expertise, possibility, and utility are figured out by $k$ decision-experts $\mathbb{Y}_{1}, \mathbb{Y}_{2}, \mathbb{Y}_{3}, \ldots, \mathbb{Y}_{k}$, with the help of $v$ crucial elements $\mathscr{X}_{1}, \mathscr{X}_{2}, \mathscr{X}_{3}, \ldots, \mathscr{X}_{v}$, treated as attributes, and let $l_{1}, l_{2}, l_{3}, \ldots, l_{v}$ be considered as the normalized weight vectors of the attributes in accordance with related MAGDM problem. Let $\lambda_{s} \in[0,1]$ be the weight of the $s$-th decision-expert $\mathbb{Y}_{s}$; therefore, the normalized weight vector for the experts is $\lambda=\left(\lambda_{1}, \lambda_{2}, \lambda_{3}, \ldots, \lambda_{k}\right)^{T}$. The strategy for complex spherical fuzzy $N$-soft-VIKOR (CSFNS -VIKOR) method is described as follows.

3.1. Complex Spherical Fuzzy N-Soft Performance Matrix. The alternatives are analyzed by decision-experts on the basis of the selected attributes and initially admeasured by numerical labels representing linguistic information relative 
to the MAGDM, such as 5 for "best," 4 for "good," 3 for "bad," and so. These numerical labels are further epitomized by $C \mathcal{S F N S} S_{f} N s$ with constraints of grading criteria, which moves us towards the $k C \mathcal{S} F N S_{f} S s$ classified by $k$ decision- experts. Furthermore, the $k C \mathcal{S} F N S_{f} S s$ regarding $k$ decision-experts are systemized in the form of complex spherical fuzzy $N$-soft performance matrices $C \mathcal{S} F N S_{f} P M s \mathscr{Z}^{s}=$ $\left(\mathscr{Z}_{b a}^{(s)}\right)_{m \times v}$ as follows:

$$
\mathscr{Z}^{s}=\left(\begin{array}{cccc}
\left(c_{1}^{1(s)}, \phi_{11}^{(s)}, \psi_{11}^{(s)}, \chi_{11}^{(s)}\right) & \left(c_{2}^{1(s)}, \phi_{12}^{(s)}, \psi_{12}^{(s)}, \chi_{12}^{(s)}\right) & \ldots & \left(c_{v}^{1(s)}, \phi_{1 v}^{(s)}, \psi_{1 v}^{(s)}, \chi_{1 v}^{(s)}\right) \\
\left(c_{1}^{2(s)}, \phi_{21}^{(s)}, \psi_{21}^{(s)}, \chi_{21}^{(s)}\right) & \left(c_{2}^{2(s)}, \phi_{22}^{(s)}, \psi_{22}^{(s)}, \chi_{22}^{(s)}\right) & \ldots & \left(c_{v}^{2(s)}, \phi_{2 v}^{(s)}, \psi_{2 v}^{(s)}, \chi_{2 v}^{(s)}\right) \\
\vdots & \vdots & \ddots & \vdots \\
\left(c_{1}^{m(s)}, \phi_{m 1}^{(s)}, \psi_{m 1}^{(s)}, \chi_{m 1}^{(s)}\right) & \left(c_{2}^{m(s)}, \phi_{m 2}^{(s)}, \psi_{m 2}^{(s)}, \chi_{m 2}^{(s)}\right) & \ldots & \left(c_{v}^{m(s)}, \phi_{m v}^{(s)}, \psi_{m v}^{(s)}, \chi_{m v}^{(s)}\right)
\end{array}\right),
$$

where $\quad \mathscr{Z}_{b a}^{(s)}=\left(\left(c_{a}^{b}\right)^{(s)}, \phi_{b a}^{(s)}, \psi_{b a}^{(s)}, \chi_{b a}^{(s)}\right)=\left(\left(c_{a}^{b}\right)^{(s)}, t_{b a}^{(s)}\right.$ $\left.e^{i 2 \pi \zeta_{b a}^{(s)}}, q_{b a}^{(s)} e^{i 2 \pi \varrho_{b a}^{(s)}}, f_{b a}^{(s)} e^{i 2 \pi \rho_{b a}^{(s)}}\right)$, and $b=\{1,2,3, \ldots, m\}, \quad a=$ $\{1,2,3, \ldots, v\}$, and $s=\{1,2,3, \ldots, k\}$ stand for the alternatives $J_{b}$, attributes $\mathscr{X}_{a}$, and decision-experts $\mathscr{Y}_{s}$, respectively.
3.2. Aggregated Complex Spherical Fuzzy N-Soft Performance Matrix. All decision-experts are adequate to manipulate the individual opinion using $C \mathcal{S} F N S_{f} W A$ operator and resulting matrix known as aggregated complex spherical fuzzy $N$-soft performance matrix is $\left(A C \mathcal{S F N S} S_{f} P M\right)$ evaluated as follows:

$$
\begin{aligned}
\mathscr{Z}_{b a} & =C \mathcal{S} F N S_{f} A_{\lambda}\left(\mathscr{Z}_{b a}^{(1)}, \mathscr{Z}_{b a}^{(2)}, \ldots, \mathscr{E}_{b a}^{(k)}\right) \\
& =\lambda_{1} \mathscr{E}_{b a}^{(1)} \oplus \lambda_{2} \mathscr{E}_{b a}^{(2)} \oplus \cdots \oplus \lambda_{s} \mathscr{E}_{b a}^{(k)} \\
& =\left(\max _{s=1}^{k}\left(c_{a}^{b}\right)^{(s)}, \sqrt{1-\prod_{s=1}^{k}\left(1-\left(t_{b a}^{(s)}\right)^{2}\right)^{\lambda_{s}} e^{i 2 \pi} \sqrt{1-\prod_{s=1}^{k}\left(1-\left(\zeta_{b a}^{(s)}\right)^{2}\right)^{\lambda_{s}}},\left(\prod_{s=1}^{k} q_{b a}^{(s)}\right) e^{i 2 \pi} \prod_{s=1}^{k} e_{b a}^{(s)}, \prod_{s=1}^{k} f_{b a}^{(s)} e^{i 2 \pi} \prod_{s=1}^{k} \rho_{b a}^{(s)}}\right) \\
& =\left(c_{a}^{b}, t_{b a} e^{i 2 \pi \zeta_{b a}}, q_{b a} e^{i 2 \pi \rho_{b a}}, f_{b a} e^{i 2 \pi \rho_{b a}}\right) .
\end{aligned}
$$

The $A C \mathcal{S F N S} S_{f} P M$ is as follows:

$$
\begin{aligned}
& \mathscr{P}=\left(\begin{array}{cccc}
\left(c_{1}^{1}, \phi_{11}, \psi_{11}, \chi_{11}\right) & \left(c_{2}^{1}, \phi_{12}, \psi_{12}, \chi_{12}\right) & \ldots & \left(c_{a}^{1}, \phi_{1 v}, \psi_{1 v}, \chi_{1 v}\right) \\
\left(c_{1}^{2}, \phi_{21}, \psi_{21}, \chi_{21}\right) & \left(c_{2}^{2}, \phi_{22}, \psi_{22}, \chi_{22}\right) & \ldots & \left(c_{a}^{2}, \phi_{2 v}, \psi_{2 v}, \chi_{2 v}\right) \\
\vdots & \vdots & \ddots & \vdots \\
\left(c_{1}^{m}, \phi_{m 1}, \psi_{m 1}, \chi_{m 1}\right) & \left(c_{2}^{m}, \phi_{m 2}, \psi_{m 2}, \chi_{m 2}\right) & \ldots & \left(c_{v}^{m}, \phi_{m v}, \psi_{m v}, \chi_{m v}\right)
\end{array}\right) . \\
& \check{\mathscr{E}}_{a}= \begin{cases}\max _{b} \mathscr{X}_{b a}, & \text { if } \mathscr{X}_{a} \in \Omega_{C}, \\
\min _{b} \mathscr{X}_{b a}, & \text { if } \mathscr{X}_{a} \in \Omega_{B},\end{cases}
\end{aligned}
$$

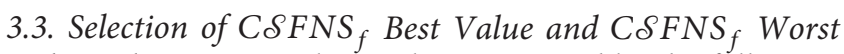
Value. The $C \mathcal{S} F N S_{f}$ best value is assessed by the following formula denoted by $\widehat{\mathscr{E}}_{a}$ :

$$
\widehat{\mathscr{E}}_{a}=\left\{\begin{array}{cl}
\max _{b} \mathscr{E}_{b a}, & \text { if } \mathscr{X}_{a} \in \Omega_{B}, \\
\min _{b} \mathscr{E}_{b a}, & \text { if } \mathscr{X}_{a} \in \Omega_{C},
\end{array}\right.
$$

where $\widehat{\mathscr{E}}_{a}=\left(\widehat{c}_{a}^{b}, \widehat{t}_{b a} e^{i 2 \pi \widehat{\zeta}_{b a}}, \widehat{q}_{b a} e^{i 2 \pi \widehat{e}_{b a}}, \widehat{f}_{b a} e^{i 2 \pi \widehat{\rho}_{b a}}\right)$.

The $C \mathcal{S F N S} S_{f}$ worst value is assessed by the following formula denoted by $\check{\mathscr{Z}}_{a}$ : where $\check{\mathscr{E}}_{a}=\left(\check{c}_{a}^{b} \check{t}_{b a} e^{i 2 \pi \check{\zeta}_{b a}}, \check{q}_{b a} e^{i 2 \pi \check{\check{c}}_{b a}}, \check{f}_{b a} e^{i 2 \pi \check{\rho}_{b a}}\right)$.

The score value $\mathbb{S}\left(\mathscr{Z}_{b a}\right)$ and accuracy value $\mathbb{A}\left(\mathscr{E}_{b a}\right)$ are utilized to compare the $C \mathcal{S} F N S_{f} N s$ in $A C \mathcal{S} F N S_{f} P M$.

3.4. Evaluating $\mathbb{S}_{b}$ and $\mathbb{R}_{b}$. The normalized Euclidean distance is utilized to evaluate the group utility measure $\mathbb{S}_{b}$ and 
individual regret measure $\mathbb{R}_{b}$ with the provision of normalized weights of attributes as follows:

$$
\begin{array}{r}
\mathbb{S}_{b}=\sum_{a=1}^{v} l_{a} \frac{d\left(\widehat{\mathscr{E}}_{a}, \mathscr{Z}_{b a}\right)}{d\left(\check{\mathscr{X}}_{a}, \mathscr{I}_{b a}\right)}, \\
\mathbb{R}_{b}=\max _{1<a<v} l_{a} \frac{d\left(\widehat{\mathscr{E}}_{a}, \mathscr{E}_{b a}\right)}{d\left(\check{\mathscr{E}}_{a}, \mathscr{E}_{b a}\right)} .
\end{array}
$$

$$
\begin{aligned}
d\left(\widehat{\mathscr{X}}_{a}, \mathscr{E}_{b a}\right)= & \left(\frac { 1 } { 4 } \left[\left(\left(\frac{\widehat{c}_{a}^{b}}{N-1}\right)^{2}-\left(\frac{c_{a}^{b}}{N-1}\right)^{2}\right)^{2}+\left(\hat{t}_{b a}^{2}-t_{b a}^{2}\right)^{2}+\left(\hat{q}_{b a}^{2}-q_{b a}^{2}\right)^{2}+\left(\widehat{f}_{b a}^{2}-f_{b a}^{2}\right)^{2}\right.\right. \\
& \left.\left.+\left(\widehat{\zeta}_{b a}^{2}-\zeta_{b a}^{2}\right)^{2}+\left(\widehat{\varrho}_{b a}^{2}-\varrho_{b a}^{2}\right)^{2}+\left(\widehat{\rho}_{b a}^{2}-\rho_{b a}^{2}\right)^{2}\right]\right)^{1 / 2}, \\
d\left(\check{\mathscr{X}}_{a}, \mathscr{E}_{b a}\right)= & \left(\frac { 1 } { 4 } \left[\left(\left(\frac{\check{c}_{a}^{b}}{N-1}\right)^{2}-\left(\frac{c_{a}^{b}}{N-1}\right)^{2}\right)^{2}+\left(\check{t}_{b a}^{2}-t_{b a}^{2}\right)^{2}+\left(\check{q}_{b a}^{2}-q_{b a}^{2}\right)^{2}+\left(\check{f}_{b a}^{2}-f_{b a}^{2}\right)^{2}\right.\right. \\
& \left.\left.+\left(\check{\zeta}_{b a}^{2}-\zeta_{b a}^{2}\right)^{2}+\left(\check{\varrho}_{b a}^{2}-\varrho_{b a}^{2}\right)^{2}+\left(\check{\rho}_{b a}^{2}-\rho_{b a}^{2}\right)^{2}\right]\right)^{1 / 2} .
\end{aligned}
$$

The optimal values of $\mathbb{S}_{b}$ and $\mathbb{R}_{b}$ are as follows:

$$
\begin{aligned}
& \widehat{\mathbb{S}}=\min _{b} \mathbb{S}_{b}, \\
& \check{\mathbb{S}}=\max _{b} \mathbb{S}_{b}, \\
& \widehat{\mathbb{R}}=\min _{b} \mathbb{R}_{b}, \\
& \check{\mathbb{R}}=\max _{b} \mathbb{R}_{b} .
\end{aligned}
$$

The $\widehat{\mathbb{S}}$ and $\widehat{\mathbb{R}}$ correspond to a maximum majority rule index and a minimum regret of opponent strategy, respectively.

3.5. Compromise Measure $\mathbb{Q}_{b}$. The configurations of utility measure and regret measure of feasible choice $J_{b}$ link up for the compromise measure $\mathbb{Q}_{b}$ as follows:

$$
\mathbb{Q}=\xi\left(\frac{\mathbb{S}_{b}-\widehat{\mathbb{S}}_{b}}{\check{\mathbb{S}}_{b}-\widehat{\mathbb{S}}_{b}}\right)+(1-\xi)\left(\frac{\mathbb{R}_{b}-\widehat{\mathbb{R}}_{b}}{\check{\mathbb{R}}_{b}-\widehat{\mathbb{R}}_{b}}\right),
$$

where $\xi \in[0,1]$ is the coefficient strategy of the majority of the attributes and in most cases taken as 0.5 , usually for the sake of equal weightage of both the configurations, $\xi=0.5$. Moreover, $\xi=1$, representing that the compromise solution is biased towards the maximum group utility. On the other hand, $\xi=0$ shows the biasness towards minimum individual regret.

3.6. Ranking of Alternatives. The values of ranking measures $\mathbb{S}_{b}, \mathbb{R}_{b}$, and $\mathbb{Q}_{b}$, corresponding to each alternative, are arranged in ascending order so that we get three ranking lists that further play an important role in finding a compromise solution. Moreover, the alternative with minimum value regarding three ranking lists is considered as the best feasible option.

3.7. Compromise Solution. For compromise solution consisting of alternative $J^{(1)}$ with a minimum value of ranking measure $\mathbb{Q}$, the following conditions should hold, which are described as follows:

$\mathbb{C}_{1}$ : acceptable advantage:

$$
\mathbb{Q}\left(J^{(2)}\right)-\mathbb{Q}\left(J^{(1)}\right) \geq \frac{1}{m-1},
$$

where $J^{(1)}$ and $J^{(2)}$ are the alternatives with the initial and subsequent position in the ranking list and $m$ representing the number of alternatives.

$\mathbb{C}_{2}$ : acceptable stability: $J^{(1)}$ should be ranked first with respect to $\mathbb{S}$ or $\mathbb{R}$. The compromise solution within the proposed method can be assumed stable in all possible situations of "voting by majority rule $(\xi>0.5)$," "by consensus $(\xi=0.5)$," or "by veto $(\xi<0.5)$."

Moreover, if the condition $\mathbb{C}_{1}$ is not satisfied, the compromise solution set contains the alternatives satisfying the following inequality:

$$
\mathbb{Q}\left(J^{(b)}\right)-\mathbb{Q}\left(J^{(1)}\right)<\frac{1}{m-1}, \quad \forall 1 \leq b \leq m .
$$

But, if condition $\mathbb{C}_{2}$ is not fulfilled, both alternatives $J^{(1)}$ and $J^{(2)}$ are known as compromise solutions of the MAGDM problem. 
The flowchart of the proposed $C \mathcal{S F N S _ { f }}$-VIKOR method is given in Figure 1.

\section{Application to Group Decision-Making}

In this section, the proposed $C \mathcal{S} F N S_{f}$-VIKOR method is carried out for the MAGDM problem in the domain of business administration. Precisely, the proposed

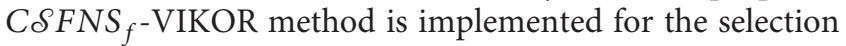
of firms for the Saudi oil refinery project in Pakistan.

4.1. Selection of Firm for the Saudi Oil Refinery Project in Pakistan. China-Pakistan Economic Corridor (CPEC) is a prudent economic project that upgrades the financial activities in Pakistan and China, serving as a gateway for the Middle East, Europe, and Africa. Specifically, there are advantageous aspects for the people of Gwadar as this project has industrious and everlasting benefits. In a sign of its enormous appliances, Saudi Arabia made a decision to establish a mega oil city in Pakistan at the spot of Gwadar and get involved in business and investment under the flagship of CPEC. A firm or company will be hired for the formation of the mega oil city, as the oil will be imported from Gulf and will be stored at the proposed Gwadar oil city, within the time period of one year. For this purpose, a team of three decision-makers $\mathbb{Y}_{1}, \mathbb{Y}_{2}$, and $\mathbb{Y}_{3}$ are chosen, and the selected concise list of five firms is as follows:

\section{$J_{1}$ : Rabigh Refining and Petrochemical firm \\ $\mathrm{J}_{2}$ : Petromin Company \\ $J_{3}$ : Saudi Aramco Total Refining and Petrochemical firm \\ $J_{4}$ : Alkhorayef Lubricants Company \\ $J_{5}$ : Yanbu Aramco Sinopec Refining Company (YAS- REF) Ltd.}

According to the proficiency and expertise of the MAGDM problem, weights for the decision-makers $\mathbb{Y}_{1}, \mathbb{Y}_{2}$, and $\mathbb{Y}_{3}$ are $0.34,0.31$, and 0.35 , respectively.

Decision-makers thoroughly study the five firms according to the requirements of the project and captured data from the websites http://www.dnb.com (Dun and Bradstreet Data Cloud) and http://www.investopedia.com, on the basis of precise characteristics or parameters discussed in Table 1.

Decision-makers assigned weight vector $(0.26,0.24,0.05,0.15,0.30)^{T}$ to the attributes taking into account the excellence and type of the attributes. For the selection of the best firm, we adopt the $C \mathcal{S} F N S_{f}$-VIKOR method as follows:

Step 1: decision-makers modeled six-soft set, arranged in Table 2, related to attributes of the MAGDM problem, where five asterisks mean "excellent performance," four asterisks mean "great performance," three asterisks mean "good performance," two asterisks mean "average performance," one asterisk means "bad performance," and big dot means "very bad performance."
The criteria for the association of the CSFNs to 6-soft sets of decision-makers is given in Table 3, so that the opinions of the decision-makers in the form of $C \mathcal{S} F N S_{f} P M s \mathscr{Z}^{1}, \mathscr{Z}^{2}$, and $\mathscr{Z}^{3}$ are arranged in Tables 4 , 5 , and 6 , simultaneously.

Step 2: the judgments of all performance matrices of decision-makers amalgamated into $A C \mathcal{S F N S} P M$ using $C \mathcal{S} F N S_{f} A_{\lambda}$ operator are summarized in Table 7.

Step 3: for the evaluation of the worst and the best values, the score values of the entries of $A C \mathcal{S} F N S_{f} P M$ are calculated, keeping in view the benefit and cost type criteria where all the attributes are benefit type except $\mathscr{X}_{3}$. The worst and best values are calculated from equations (24) and (25) that are shown in Table 8.

Step 4: the normalized Euclidean distance is formulated by equations (28) and (29), which is utilized to measure the separation of best value from each entry of $\operatorname{CSFNS}_{f} P M$ and also from worst value simultaneously, organized in Table 9. These distances are further employed in equations (26) and (27) to investigate the group utility measure $\mathbb{S}$ and individual regret measure $\mathbb{R}$, calculated in Table 10 .

Step 5: the compromise measure $\mathbb{Q}_{b}$ related to each alternative or firm, with $\xi=0.50$, is estimated through equation (31) and pinned up in Table 10.

Step 6: furthermore, the alternatives are ranked on the basis of the group utility, regret measure, and compromise measure that is illuminated in Table 11.

The firm $J_{3}$ has rank order 1 on the basis of the compromise measure $\mathbb{Q}$, and also, the remaining two conditions of SSFNS $_{f}$-VIKOR method are satisfied and calculated as follows:

(1) $\mathbb{Q}\left(J_{4}\right)-\mathbb{Q}\left(J_{3}\right)=0.5559-0=0.5559 \geq(1 / 6-1)=0.2$

(2) $J_{3}$ is the best firm regarding $\mathbb{S}$ and $\mathbb{R}$, and ranking orders are $J_{3}>J_{4}>J_{5}>J_{1}>J_{2}$

Thus, the firm $J_{3}$ is best for the establishment of the Saudi oil refinery project in Pakistan.

\section{Sensitivity Test}

In the sensitivity test, the role of coefficient strategy $\xi$ playing as the weight of group utility measure and $1-\xi$ as the weight of individual regret measure is investigated to demonstrate

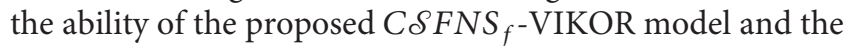
stability of the solution, which is evaluated by applying the presented methodology.

$\xi$ is assigned different values from the unit interval $[0,1]$, and then we analyze the strength and potency of the computed decisions from the proposed methodology. In this case, it is assumed that the variation in the coefficient strategy weight occurs because the MAGDM panel can prioritize both (group utility or individual regret) measures, according to the future necessities of the proposed case study. However, $\xi \in[0.6,1]$ illustrates that the compromise solution is subjective to maximum group utility, and 


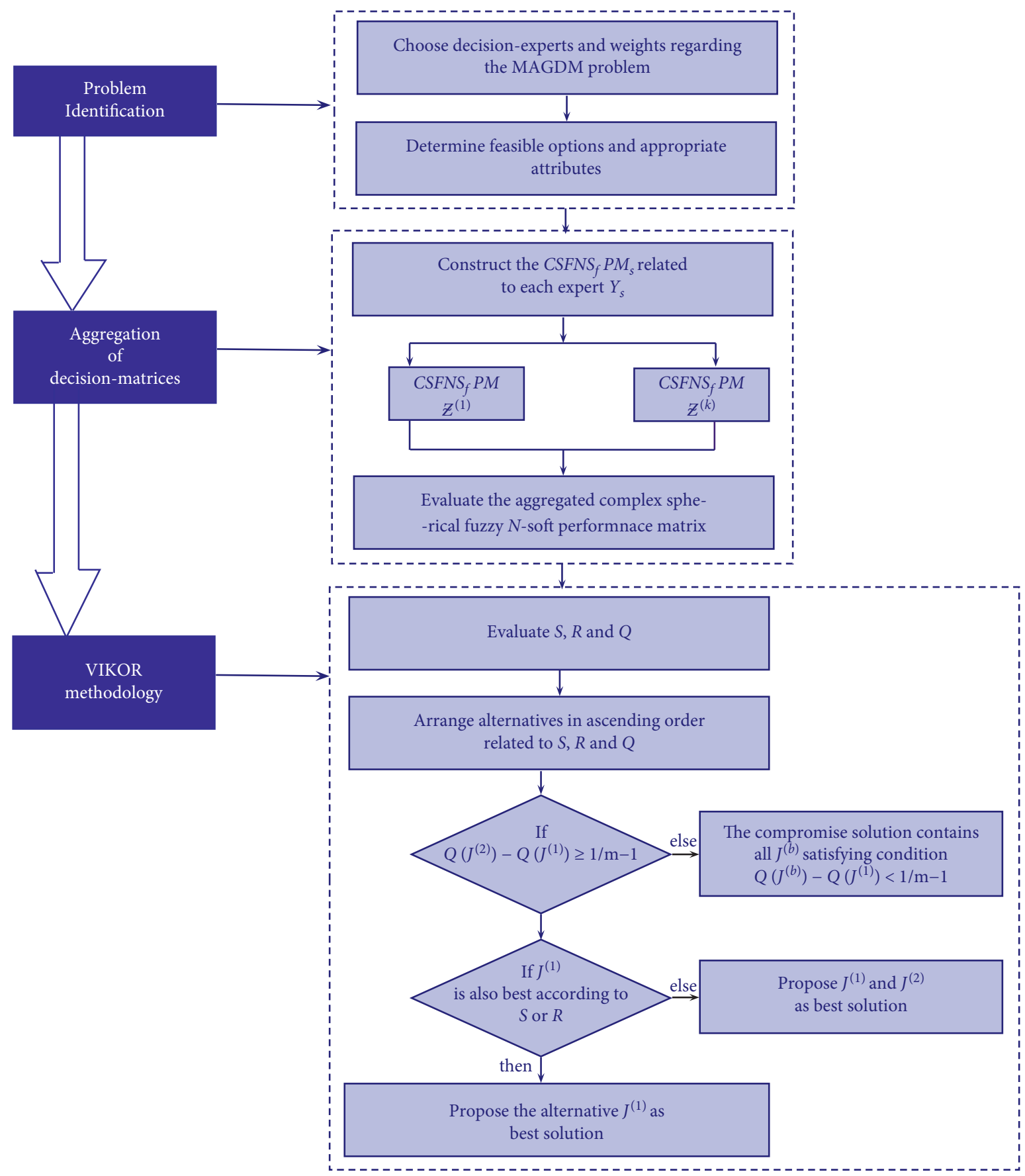

Figure 1: Flowchart of the $C \mathcal{S} F N S_{f}$-VIKOR method.

$\xi \in[0,0.4]$ represents the biasness towards minimum individual regret.

Figure 2 illustrate that the variation in $\xi$ from 0 to 1 affects the value of the compromise measure $\mathbb{Q}$ of alternatives, but the ranking order of the alternatives and the best solution (i.e., $J_{3}$ ) are static in all cases.

\section{Comparative Analysis}

This section elaborates the superiority and caliber of the proposed CSFNS -VIKOR method by solving the proposed MAGDM problem "Selection of firm for Saudi oil refinery project in Pakistan" by complex spherical fuzzyVIKOR ( $C \mathcal{S} F$-VIKOR) method, proposed by Akram et al. [33]. For the selection of the best firm, we adopt the $C \mathcal{S F}$-VIKOR method as follows:

Step 1: the initial judgments of the decision-makers are in the form of grade level as shown in Table 2 that further transformed into $C \mathcal{S} F N s$, following the grading criteria defined in Table 3 . The personal assessment of the decision-makers related to the parameters are denoted by $C \mathcal{S} F N S_{f} P M s\left(\mathscr{Z}^{1}, \mathscr{Z}^{2}\right.$, and $\mathscr{Z}^{3}$ ) and arranged in Tables 12,13 , and 14 respectively. 
TABLE 1: Study of attributes for the MAGDM problem.

\begin{tabular}{|c|c|c|c|}
\hline$x_{1}$ & $\begin{array}{l}\text { Strong risk } \\
\text { Management }\end{array}$ & It is imperative to select a business firm that is financially strong and fully endorsed. & $\begin{array}{l}\text { Benefit } \\
\text { type }\end{array}$ \\
\hline$x_{2}$ & Modern technology: & $\begin{array}{c}\text { It includes the latest apparatus and machinery, up-to-date software, and other high-tech } \\
\text { innovations. }\end{array}$ & $\begin{array}{l}\text { Benefit } \\
\text { type }\end{array}$ \\
\hline$x_{3}$ & Cost price & $\begin{array}{l}\text { This attribute includes initial cost, maintenance cost, and production cost. The firm with the } \\
\text { lowest cost is preferable. }\end{array}$ & Cost type \\
\hline$x_{4}$ & Plan and vision & It is necessary to choose a firm whose strategy related to the project is impactful and profitable. & $\begin{array}{l}\text { Benefit } \\
\text { type }\end{array}$ \\
\hline$x_{5}$ & Experience & $\begin{array}{l}\text { It includes business-related experience, knowledge, and expertise with fluctuating track record } \\
\text { and success. }\end{array}$ & $\begin{array}{l}\text { Benefit } \\
\text { type }\end{array}$ \\
\hline
\end{tabular}

TABle 2: Decision-makers opinions regarding attributes.

\begin{tabular}{|c|c|c|c|c|}
\hline Attributes & Alternatives & $\mathbb{Y}_{1}$ & $\mathbb{Y}_{2}$ & $\mathbb{Y}_{3}$ \\
\hline$X_{1}$ & $\begin{array}{l}J_{1} \\
J_{2} \\
J_{3} \\
J_{4} \\
J_{5}\end{array}$ & $\begin{array}{c}*=1 \\
*=1 \\
* * * * *=5 \\
* * * *=4 \\
* * *=3\end{array}$ & $\begin{array}{c}* *=2 \\
\cdot=0 \\
* * * *=4 \\
* * *=3 \\
* *=2\end{array}$ & $\begin{array}{c}\cdot=0 \\
*=1 \\
* * * * *=5 \\
* * * *=4 \\
* * *=3\end{array}$ \\
\hline$x_{2}$ & $\begin{array}{l}J_{1} \\
J_{2} \\
J_{3} \\
J_{4} \\
J_{5}\end{array}$ & $\begin{array}{c}\cdot=0 \\
*=1 \\
* * * *=4 \\
* * *=3 \\
* *=2\end{array}$ & $\begin{array}{c}*=1 \\
*=1 \\
* * * * *=5 \\
* * * *=4 \\
* * *=3\end{array}$ & $\begin{array}{c}\cdot=0 \\
*=1 \\
* * * * *=5 \\
* * * *=4 \\
* * *=3\end{array}$ \\
\hline $\mathscr{X}_{3}$ & $\begin{array}{l}J_{1} \\
J_{2} \\
J_{3} \\
J_{4} \\
J_{5} \\
\end{array}$ & $\begin{array}{c}\cdot=0 \\
\cdot=0 \\
* * * *=4 \\
* * * *=4 \\
* * *=3\end{array}$ & $\begin{array}{c}\cdot=0 \\
*=1 \\
* * * * *=5 \\
* * * * *=5 \\
* * *=3\end{array}$ & $\begin{array}{c}\cdot=0 \\
*=1 \\
* * * * *=5 \\
* * * *=4 \\
* * *=3\end{array}$ \\
\hline$x_{4}$ & $\begin{array}{l}J_{1} \\
J_{2} \\
J_{3} \\
J_{4} \\
J_{5} \\
\end{array}$ & $\begin{array}{c}\cdot=0 \\
* *=2 \\
* * * * *=5 \\
* * * *=4 \\
* * *=3\end{array}$ & $\begin{array}{c}\cdot=0 \\
*=1 \\
* * * * *=5 \\
* * *=3 \\
* * *=3\end{array}$ & $\begin{array}{c}*=1 \\
* *=2 \\
* * * *=4 \\
* * * * *=5 \\
* *=2\end{array}$ \\
\hline$X_{5}$ & $\begin{array}{l}J_{1} \\
J_{2} \\
J_{3} \\
J_{4} \\
J_{5}\end{array}$ & $\begin{array}{c}*=1 \\
*=1 \\
* * * * *=5 \\
* * * *=4 \\
* * *=3\end{array}$ & $\begin{array}{c}\cdot=0 \\
*=1 \\
* * * * *=5 \\
* * * *=4 \\
* * *=3\end{array}$ & $\begin{array}{c}\cdot=0 \\
*=1 \\
* * * * *=5 \\
* * * *=4 \\
* * *=3\end{array}$ \\
\hline
\end{tabular}

TABLE 3: Grading criteria for CSF6SS.

\begin{tabular}{lcccccc}
\hline $\begin{array}{l}h_{z}^{w} / J \\
\text { Grades }\end{array}$ & \multicolumn{2}{c}{ Degree of yes } & \multicolumn{2}{c}{ Neutral membership } & \multicolumn{2}{c}{ Degree of no } \\
\hline$t_{b a}$ & $2 \pi \zeta_{b a}$ & $q_{b a}$ & $2 \pi \varrho_{b a}$ & $f_{b a}$ & $2 \pi \rho_{b a}$ \\
\hline$c_{a}^{b}=0$ & & {$[0,0.30 \pi)$} & {$[0,0.0170)$} & {$[0,0.0340 \pi)$} & $(0.90,1.00]$ & {$[1.80 \pi, 2.00 \pi]$} \\
\hline$c_{a}^{b}=1$ & {$[0.15,0.30)$} & {$[0.30 \pi, 0.60 \pi)$} & {$[0.0170,0.0819)$} & {$[0.0340 \pi, 0.1638 \pi)$} & $(0.75,0.90]$ & {$[1.50 \pi, 1.80 \pi)$} \\
\hline$c_{a}^{b}=2$ & {$[0.30,0.50)$} & {$[0.60 \pi, 1.00 \pi)$} & {$[0,0.0170)$} & $[0,0.0340 \pi))$ & $(0.50,0.75]$ & {$[1.00 \pi, 1.50 \pi)$} \\
\hline$c_{a}^{b}=3$ & {$[0.50,0.75)$} & {$[1.00 \pi, 1.50 \pi)$} & {$[0.0170,0.0819)$} & {$[0.0340 \pi, 0.1638 \pi)$} & $(0.30,0.50]$ & {$[0.60 \pi, 1.00 \pi)$} \\
\hline$c_{a}^{b}=4$ & {$[0.75,0.90)$} & {$[1.50 \pi, 1.80 \pi)$} & {$[0,0.0170)$} & $[0,0.0340 \pi))$ & $(0.15,0.30]$ & {$[0.30 \pi, 0.60 \pi)$} \\
\hline$c_{a}^{b}=5$ & {$[0.90,1.00]$} & {$[1.80 \pi, 2.00 \pi]$} & {$[0.0170,0.0819)$} & {$[0.0340 \pi, 0.1638 \pi)$} & {$[0,0.15]$} & {$[0,0.30 \pi)$} \\
\hline
\end{tabular}




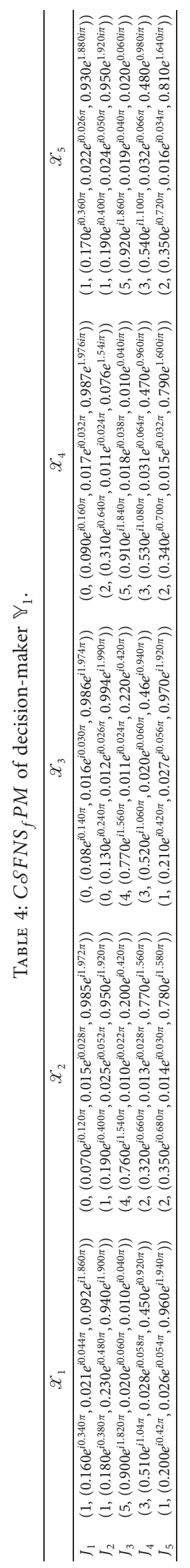




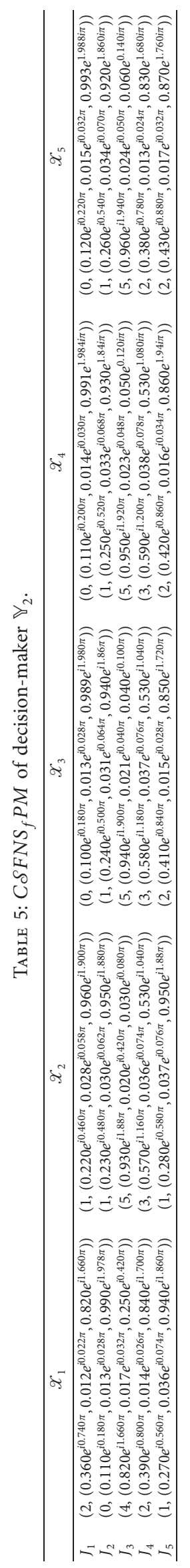




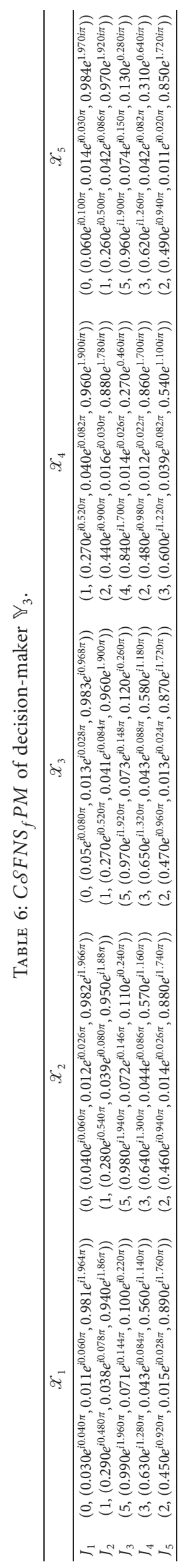




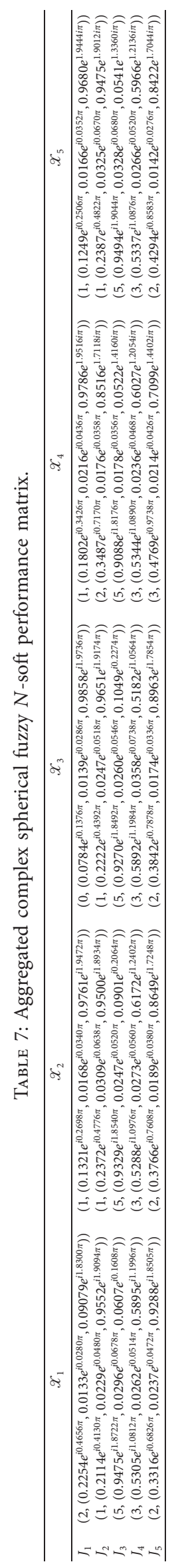


TABLE 8: $C \mathcal{S} F N S_{f}$ best and worst values related to the attributes.

\begin{tabular}{llc}
\hline Attribute & Best value & Worst value \\
\hline$X_{1}$ & $\left(5,\left(0.9475 e^{i 1.8722 \pi}, 0.0296 e^{i 0.0678 \pi}, 0.0607 e^{i 0.1608 \pi}\right)\right)$ & $\left(1,\left(0.2114 e^{i 0.4130 \pi}, 0.0229 e^{i 0.0480 \pi}, 0.9552 e^{i 1.9094 \pi}\right)\right)$ \\
$\mathscr{X}_{2}$ & $\left(5,\left(0.9329 e^{i 1.8540 \pi}, 0.0247 e^{i 0.0520 \pi}, 0.0901 e^{i 0.2064 \pi}\right)\right)$ & $\left(1,\left(0.1321 e^{i 0.2698 \pi}, 0.0168 e^{i 0.0340 \pi}, 0.9761 e^{i 1.9472 \pi}\right)\right)$ \\
$\mathscr{X}_{3}$ & $\left(0,\left(0.0784 e^{i 0.1376 \pi}, 0.0139 e^{i 0.0286 \pi}, 0.9858 e^{i 1.9736 \pi}\right)\right)$ & $\left(5,\left(0.9270 e^{i 1.8492 \pi}, 0.0260 e^{i 0.0546 \pi}, 0.1049 e^{i 0.2274 \pi}\right)\right)$ \\
$\mathscr{X}_{4}$ & $\left(5,\left(0.9088 e^{i 1.8176 \pi}, 0.0178 e^{i 0.0356 \pi}, 0.0522 e^{1.4160 i \pi}\right)\right)$ & $\left(1,\left(0.1802 e^{i 0.3426 \pi}, 0.0216 e^{i 0.0436 \pi}, 0.9786 e^{1.9516 i \pi}\right)\right)$ \\
$\mathscr{X}_{5}$ & $\left(5,\left(0.9494 e^{i 1.9044 \pi}, 0.0328 e^{i 0.0680 \pi}, 0.0541 e^{1.3360 i \pi}\right)\right)$ & $\left(1,\left(0.1249 e^{i 0.2506 \pi}, 0.0166 e^{i 0.0352 \pi}, 0.9680 e^{1.9444 i \pi}\right)\right)$ \\
\hline
\end{tabular}

TABle 9: Normalized Euclidean distance.

\begin{tabular}{|c|c|c|c|c|c|}
\hline Alternative & $d\left(\widehat{\mathscr{X}}_{1}, \mathscr{Z}_{b 1}\right)$ & $d\left(\hat{\mathscr{Z}}_{2}, \mathscr{Z}_{b 2}\right)$ & $d\left(\widehat{\mathscr{X}}_{3}, \mathscr{Z}_{b 3}\right)$ & $d\left(\hat{\mathscr{E}}_{4}, \mathscr{Z}_{b 4}\right)$ & $d\left(\widehat{\mathscr{X}}_{5}, \mathscr{Z}_{b 5}\right)$ \\
\hline$J_{1}$ & 0.9304 & 0.9660 & 0.00049 & 1.0035 & 1.0316 \\
\hline$J_{2}$ & 0.9986 & 0.9124 & 0.0497 & 0.8319 & 0.9954 \\
\hline$J_{3}$ & 0 & 0 & 1.0362 & 0 & 0 \\
\hline$J_{4}$ & 0.5860 & 0.5596 & 0.5806 & 0.5628 & 0.5957 \\
\hline$J_{5}$ & 0.9169 & 0.7890 & 0.1785 & 0.6430 & 0.8308 \\
\hline
\end{tabular}

TABLE 10: The values of $\mathbb{S}_{b}, \mathbb{R}_{b}$, and $\mathbb{Q}_{b}$.

\begin{tabular}{lccc}
\hline Alternative & $\mathbb{S}_{b}$ & $\mathbb{R}_{b}$ & $\mathbb{Q}_{b}$ \\
\hline$J_{1}$ & 0.9322 & 0.2422 & 0.9014 \\
$J_{2}$ & 0.9029 & 0.2894 & 0.9833 \\
$J_{3}$ & 0.05 & 0.05 & 0 \\
$J_{4}$ & 0.5769 & 0.1732 & 0.5559 \\
$J_{5}$ & 0.7810 & 0.2416 & 0.7418 \\
\hline
\end{tabular}

TABLE 11: Ranking of firms.

\begin{tabular}{lccccc}
\hline Alternatives & $J_{1}$ & $J_{2}$ & $J_{3}$ & $J_{4}$ & \\
\hline Ranking order of $\mathbb{S}$ & 5 & 4 & 1 & 2 & 3 \\
Ranking order of $\mathbb{R}$ & 5 & 4 & 1 & 2 & 3 \\
Ranking order of $\mathbb{Q}$ & 5 & 4 & 1 & 2 & 3 \\
\hline
\end{tabular}

Step 2: the aggregated complex spherical fuzzy performance matrix $A C S F P M$ securing the collective opinion of the decision-makers through a complex spherical fuzzy weighted average $(C \mathcal{S F W} A)$ operator is employing as follows:

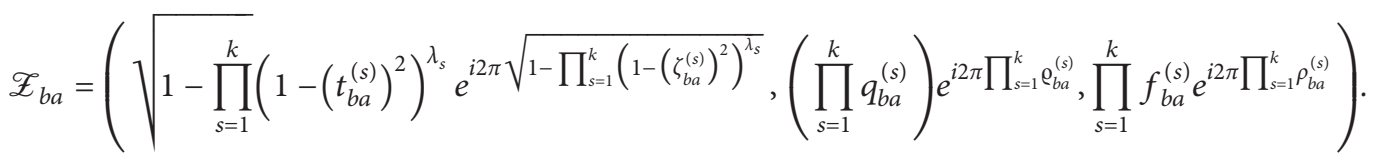

The ACSFPM shown in Table 15.

Step 3: furthermore, for the evaluation of worst and best attributes in ACSFPM, equations (24) and (25) are brought into play, despite that the comparison of complex spherical fuzzy numbers in $A C S F P M$ is computed by exploiting the score function of each entry as follows:

$$
\operatorname{Sc}\left(\mathscr{Z}_{b a}\right)=\left[t_{b a}^{2}-q_{b a}^{2}-f_{b a}^{2}\right]+\left[\zeta_{b a}^{2}-\varrho_{b a}^{2}-\rho_{b a}^{2}\right] .
$$

CSF's best and worst values related to the attributes are given in Table 16.

Step 4: the group utility measure $\mathbb{S}$ and individual regret measure $\mathbb{R}$ of each alternative $J_{b}$ are valuated through equations (26) and (27) in the light of 


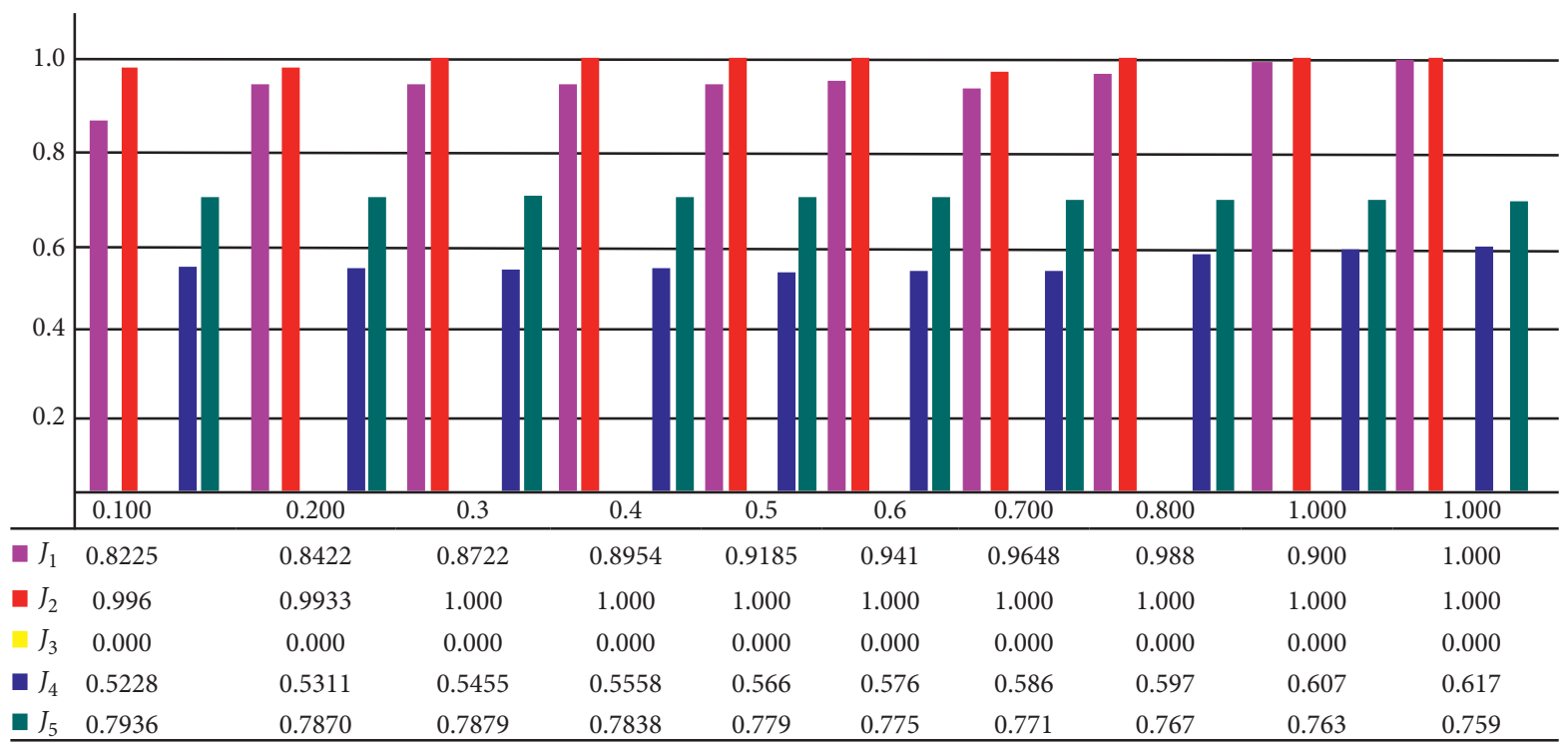

Figure 2: Sensitivity test.

normalized distance measures, indicated in the following equations and worthy attributes.

$$
\begin{aligned}
& d\left(\widehat{\mathscr{E}}_{a}, \mathscr{E}_{b a}\right)=\sqrt{\left(\frac{1}{3}\left[\left(\hat{t}_{b a}^{2}-t_{b a}^{2}\right)^{2}+\left(\widehat{q}_{b a}^{2}-q_{b a}^{2}\right)^{2}+\left(\hat{f}_{b a}^{2}-f_{b a}^{2}\right)^{2}+\left(\widehat{\zeta}_{b a}^{2}-\zeta_{b a}^{2}\right)^{2}+\left(\widehat{\varrho}_{b a}^{2}-\varrho_{b a}^{2}\right)^{2}+\left(\hat{\rho}_{b a}^{2}-\rho_{b a}^{2}\right)^{2}\right]\right)}, \\
& d\left(\check{\mathscr{E}}_{a}, \mathscr{E}_{b a}\right)=\sqrt{\left(\frac{1}{3}\left[\left(\check{t}_{b a}^{2}-t_{b a}^{2}\right)^{2}+\left(\check{q}_{b a}^{2}-q_{b a}^{2}\right)^{2}+\left(\check{f}_{b a}^{2}-f_{b a}^{2}\right)^{2}+\left(\check{\zeta}_{b a}^{2}-\zeta_{b a}^{2}\right)^{2}+\left(\check{\varrho}_{b a}^{2}-\varrho_{b a}^{2}\right)^{2}+\left(\check{\rho}_{b a}^{2}-\rho_{b a}^{2}\right)^{2}\right]\right)} .
\end{aligned}
$$

The normalized distance in equations (36) and (37) is symmetric as well as it gives positive values corresponding to each entry in $A C \mathcal{S} F P M$, listed in Table 17. In addition to that, the group utility measure $\mathbb{S}$, individual regret measure $\mathbb{R}$ and compromise ranking $\mathbb{Q}$ (around with equation (31) and $\xi=0.50$ ) are calculated in Table 18.

Step 5: following the values of $\mathbb{S}, \mathbb{R}$, and $\mathbb{Q}$ from Table 1 in ascending order, the priority order of alternatives is presented in Table 19.

Step 6: the firm $J_{3}$ has rank order one on the basis of the compromise measure $\mathbb{Q}$, and also, the remaining two conditions of the $C \delta \mathcal{S}$-VIKOR method are satisfied and calculated as follows:

(1) $\mathbb{Q}\left(J_{4}\right)-\mathbb{Q}\left(J_{3}\right)=0.5262-0=0.5262 \geq(1 / 6-1)=$ 0.2

(2) $J_{3}$ is best firm regarding $\mathbb{S}$ and $\mathbb{R}$

Thus, the firm $J_{3}$ is also the best feasible choice for the establishment of the Saudi oil refinery project in Pakistan with respect to $C \mathscr{S} F$-VIKOR method.

6.1. Discussion. The comparison provides the following results:
(1) The compromise solution assessed by the $C \mathcal{S F N S} S_{f}$-VIKOR and $C \mathcal{S F}$-VIKOR methods is the same exact, as indicated in Table 19, which implies that $J_{3}$ is the best firm for the development of the Saudi oil refinery at the place of Gwadar. Together with the same results, the rankings of the alternatives in both methods are unvarying. So, we can claim that the outcomes of the proposed $C \mathcal{S} F N S_{f}$-VIKOR method are equivalent to the $C \mathcal{S} F$-VIKOR method. The correlation between $C \delta F N S_{f}$-VIKOR and $C \mathcal{S F}$-VIKOR methods is illustrated in Table 20, and their graphical representations are put forward in Figure 3.

(2) We also implement the techniques of $\delta F$-VIKOR [27] and fuzzy-VIKOR [8] on the proposed MAGDM problem. The transparency and accuracy in the outcomes improve and glorify the level of trust and confidence about the proposed method. The comparison results are pinned up in Table 21.

(3) The comparative study of the CSFNS -VIKOR $^{-}$ method with the existing techniques demonstrates and certifies the superiority of the proposed method as fuzzy-VIKOR, $\delta F$-VIKOR, and $C \delta \mathcal{S F}$-VIKOR methods have some imperfections and limitations 


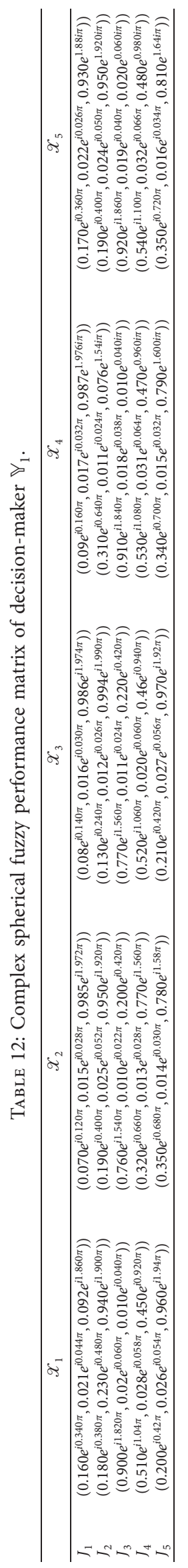




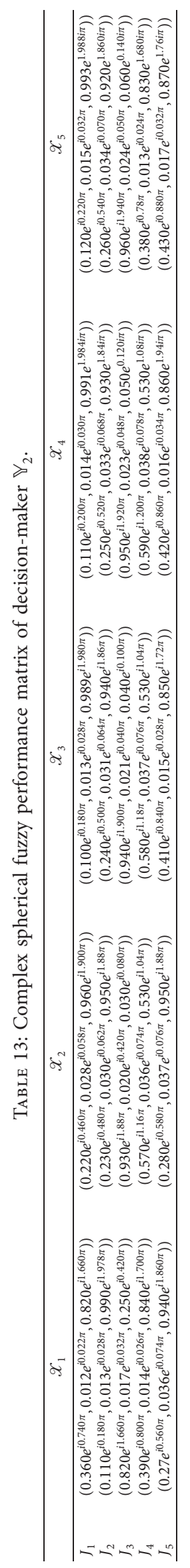




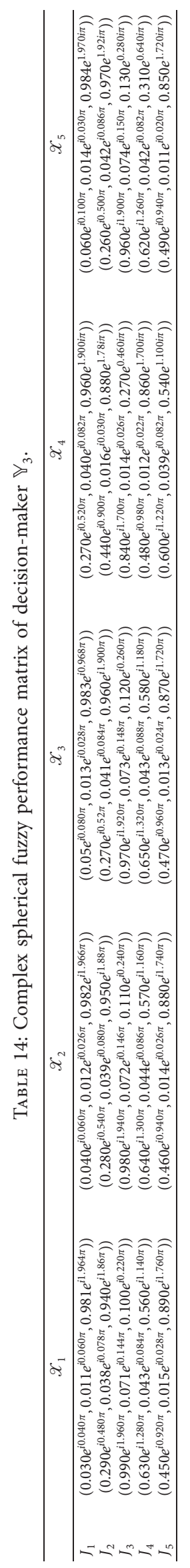




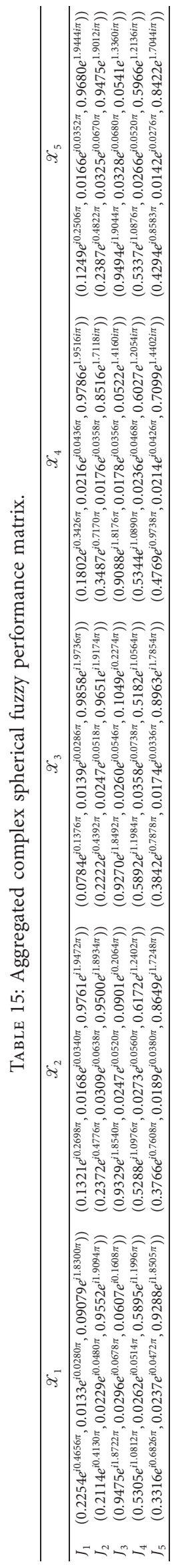


TABLE 16: $C \mathcal{S F}$ best and worst values related to the attributes.

\begin{tabular}{llc}
\hline Attribute & Best value & Worst value \\
\hline$X_{1}$ & $\left.\left(0.9475 e^{i 1.8722 \pi}, 0.0296 e^{i 0.0678 \pi}, 0.0607 e^{i 0.1608 \pi}\right)\right)$ & $\left.\left(0.2114 e^{i 0.4130 \pi}, 0.0229 e^{i 0.0480 \pi}, 0.9552 e^{i 1.9094 \pi}\right)\right)$ \\
$\mathscr{X}_{2}$ & $\left.\left(0.9329 e^{i 1.8540 \pi}, 0.0247 e^{i 0.0520 \pi}, 0.0901 e^{i 0.2064 \pi}\right)\right)$ & $\left.\left(0.1321 e^{i 0.2698 \pi}, 0.0168 e^{i 0.0340 \pi}, 0.9761 e^{i 1.9472 \pi}\right)\right)$ \\
$\mathscr{X}_{3}$ & $\left.\left(0.0784 e^{i 0.1376 \pi}, 0.0139 e^{i 0.0286 \pi}, 0.9858 e^{i 1.9736 \pi}\right)\right)$ & $\left.\left(0.9270 e^{i 1.8492 \pi}, 0.0260 e^{i 0.0546 \pi}, 0.1049 e^{i 0.2274 \pi}\right)\right)$ \\
$X_{4}$ & $\left.\left(0.9088 e^{i 1.8176 \pi}, 0.0178 e^{i 0.0356 \pi}, 0.0522 e^{1.4160 i \pi}\right)\right)$ & $\left.\left(0.1802 e^{i 0.3426 \pi}, 0.0216 e^{i 0.0436 \pi}, 0.9786 e^{1.9516 i \pi}\right)\right)$ \\
$\mathscr{X}_{5}$ & $\left.\left(0.9494 e^{i 1.9044 \pi}, 0.0328 e^{i 0.0680 \pi}, 0.0541 e^{1.3360 i \pi}\right)\right)$ & $\left.\left(0.1249 e^{i 0.2506 \pi}, 0.0166 e^{i 0.0352 \pi}, 0.9680 e^{1.9444 i \pi}\right)\right)$ \\
\hline
\end{tabular}

TABLE 17: Differences of alternatives from best values of ACSFPM.

\begin{tabular}{lccccc}
\hline Alternative & $d\left(\hat{\mathscr{E}}_{1}, \mathscr{E}_{b 1}\right)$ & $d\left(\hat{\mathscr{E}}_{2}, \mathscr{E}_{b 2}\right)$ & $d\left(\hat{\mathscr{E}}_{3}, \mathscr{E}_{b 3}\right)$ & $d\left(\hat{\mathscr{E}}_{4}, \mathscr{E}_{b 4}\right)$ & 1.0175 \\
\hline$J_{1}$ & 0.9585 & 0.9680 & 0.00005 & 0.8292 & 0 \\
$J_{2}$ & 1.0111 & 0.8960 & 0.0526 & 0 & 1.0544 \\
$J_{3}$ & 0 & 0 & 1.0480 & 0.5346 & 0 \\
$J_{4}$ & 0.5668 & 0.5302 & 0.6371 & 0.6441 & 0.5803 \\
$J_{5}$ & 0.9412 & 0.7714 & 0.1844 & $\left.\mathscr{E}_{b 5}\right)$ \\
\hline
\end{tabular}

TABle 18: The values of $\mathbb{S}_{b}, \mathbb{R}_{b}$, and $\mathbb{Q}_{b}$.

\begin{tabular}{lccc}
\hline$J_{1}$ & 0.9364 & 0.2464 & 0.9152 \\
\hline$J_{2}$ & 0.98934 & 0.2865 & 0.9757 \\
$J_{3}$ & 0.05 & 0.05 & 0 \\
$J_{4}$ & 0.5515 & 0.1651 & 0.5262 \\
$J_{5}$ & 0.7725 & 0.2420 & 0.81346 \\
\hline
\end{tabular}

TABle 19: Ranking of firms with respect to $C \mathcal{S F}$-VIKOR.

\begin{tabular}{lccccc}
\hline Alternatives & $J_{1}$ & $J_{2}$ & $J_{3}$ & $J_{4}$ & $J_{5}$ \\
\hline Ranking order of $\mathbb{S}$ & 5 & 4 & 1 & 2 & 3 \\
Ranking order of $\mathbb{R}$ & 5 & 4 & 1 & 2 & 3 \\
Ranking order of $\mathbb{Q}$ & 5 & 4 & 1 & 2 & 3 \\
\hline
\end{tabular}

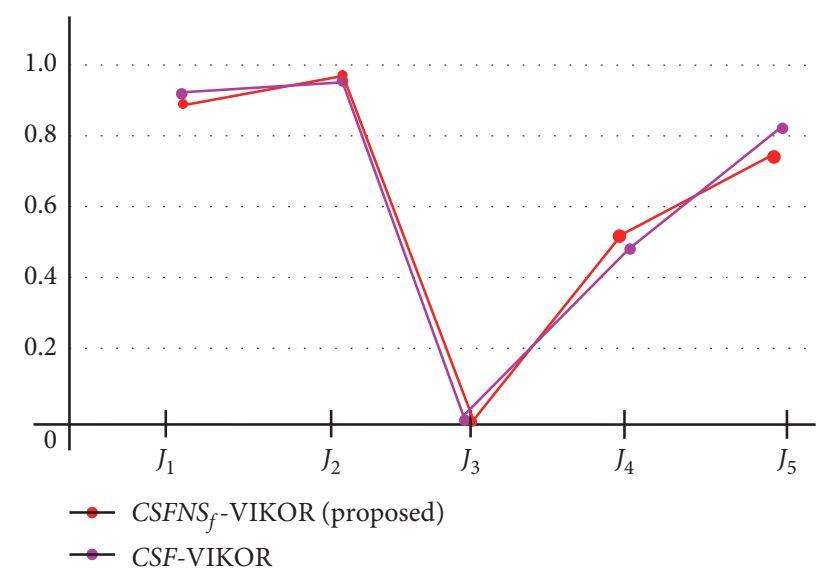

Figure 3: Comparative analysis.

that they all are impotent to understand the grades level with parameterized figures of such contemporary MAGDM problems.
TABle 20: Comparison with $C \mathcal{S} F$-VIKOR.

\begin{tabular}{lcc}
\hline Method & Ranking of firms & Best firm \\
\hline$C \mathcal{S F N S}$-VIKOR (proposed) & $J_{3}>J_{4}>J_{5}>J_{1}>J_{2}$ & $J_{3}$ \\
$C \mathcal{S F}$-VIKOR [33] & $J_{3}>J_{4}>J_{5}>J_{1}>J_{2}$ & $J_{3}$ \\
\hline
\end{tabular}

TABLE 21: Comparison with $\mathcal{S F}$-VIKOR and fuzzy-VIKOR.

\begin{tabular}{lcc}
\hline Method & Ranking of firms & Best firm \\
\hline CSFNS SIIKOR (proposed) & $J_{3}>J_{4}>J_{5}>J_{1}>J_{2}$ & $J_{3}$ \\
$\mathcal{S F}$-VIKOR [27] & $J_{3}>J_{4}>J_{5}>J_{1}>J_{2}$ & $J_{3}$ \\
Fuzzy-VIKOR [8] & $J_{3}>J_{4}>J_{5}>J_{1}>J_{2}$ & $J_{3}$ \\
\hline
\end{tabular}

\section{Advantages of Proposed Method}

(i) The principle of the VIKOR method relies on group utility measure and individual regret measure of each feasible choice, obtained using the normalized weighted vector of attributes within the normalized Euclidean distances, compromise the effects of grades level along with the two-dimensional frame.

(ii) Decision-making using the VIKOR method provides compromise solution in close proximity of the ideal solution in conjunction with a two-dimensional fusion of $C \mathcal{S F N S}$ model that enjoys four opinions of true, neutral, false, and level of the alternatives in reference to the soft information. Moreover, by keeping the level of the alternatives zero, two-dimensional information of four perspectives diversified into the $C \mathcal{S F}$ data that can be readily managed by the presented model.

(iii) The proposed strategic model even accommodates one-dimensional MAGDM problems within the ambit of spherical fuzzy information and picture fuzzy information by exerting periodic terms and ordered grades zero and raises the assurance of the proposed method via the accuracy of the results. 
(iv) Additionally, the proficiency about the neutral part elongates the presented model as the abstraction of the fuzzy $N$-soft models including intuitionistic fuzzy N-soft, Pythagorean fuzzy N-soft, and complex Pythagorean fuzzy $N$-soft environments.

\section{Conclusion}

Many real MAGDM problems have a complex pattern. A hybrid decision-making model has been introduced in this paper, which is based on the VIKOR method but allows us to solve problems posed in the elaborate form of complex spherical fuzzy $N$-soft sets. This model was developed by Akram et al. [34]. An advantage of decision-making within the two-dimensional frame of $C \mathcal{S F N S} S s$ is that the experts are free to use four conjectures of true, neutral, false, and level of the alternatives in reference to the soft information. The proposed $C \mathcal{S} F N S_{f}$-VIKOR method has been in discussion corresponding to the conflicting criteria under different methodologies. The linear normalized weights of the attributes and normalized Euclidean distances have been interpolated together, for the sake of two main features of the acclaimed VIKOR methodology, known as maximum group utility and minimum individual regret. Moreover, the coefficient of weight strategy pertaining to majority opinions and minimum regret of opponents have been exhibited for the compromise measure (ranking function). Furthermore, by keeping the level of the alternatives at zero, two-dimensional information comprising four conjectures becomes $C S F$ knowledge that can therefore be embedded in the proposed model.

The applicability and adequacy of the proposed method have been exemplified through a MAGDM problem whereupon a feasible firm is required for the development of the Saudi oil refinery project in Pakistan. For the imple-

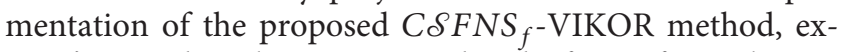
perts' inputs have been supported in the form of a nonbinary evaluation system. The $C \mathcal{S} F N S_{f} W A$ operator has been deployed for the construction of $A C \mathcal{S} F N S_{f} P M$, and score degree has been established for the comparison of two complex entities in $A C \mathcal{S} F N S_{f} P M$. In the end, a comparison has been executed, which strengthens the presented method through the results' transparency. The one-dimensional MAGDM problems under the auspices of spherical fuzzy information and picture fuzzy information can be conciliated with this structure by setting phase terms and ordered grades at zero. This speaks for the strength and validity of the presented approach. The proposed model, in accordance with neutral information, constitutes a generalization of fuzzy $N$-soft knowledge including intuitionistic fuzzy $N$-soft, Pythagorean fuzzy $N$-soft, and complex Pythagorean fuzzy $N$-soft data.

For future direction, the $C \mathcal{S} F N S_{f}$-VIKOR method can be employed for selections in other types of MAGDM problems, including solar panel selection, sites for hotel business selection, in the field of medical and sustainable suppliers. Moreover, the limitations of the proposed model invite us to formulate new paradigms. For instance, the CSFNS $_{f}$-VIKOR method is deficient to settle conditions in which the sum of squares of truth membership, neutral membership, and falsity membership of amplitude terms (or phase terms) exceeds 1 . Therefore, we can work for establishing a complex $T$-spherical fuzzy $N$-soft-VIKOR methodology to extend the current boundary constraints. Moreover, a computer program can be created to handle the difficulties that appear in the presence of large numbers of alternatives and attributes.

\section{Data Availability}

No data were used to support this study.

\section{Conflicts of Interest}

The authors declare that they have no conflicts of interest.

\section{Acknowledgments}

This project was funded by the Deanship of Scientific Research (DSR), King Abdulaziz University, Jeddah, under grant no. D1442-325-130. The authors, therefore, gratefully acknowledge DSR technical and financial support.

\section{References}

[1] S. Opricovic, Multicriteria optimization of civil engineering systems, vol. 2, no. 1, , pp. 5-21, Faculty Civil Engineering, Belgrade, Serbia, 1998, Ph.D. thesis.

[2] S. Opricovic and G.-H. Tzeng, "Compromise solution by MCDM methods: a comparative analysis of VIKOR and TOPSIS," European Journal of Operational Research, vol. 156, no. 2, pp. 445-455, 2004.

[3] A. Yazdani-Chamzini, M. M. Fouladgar, E. K. Zavadskas, and S. H. H. Moini, "Selecting the optimal renewable energy using multi criteria decision making," Journal of Business Economics and Management, vol. 14, no. 5, pp. 957-978, 2013.

[4] L. A. Zadeh, "Fuzzy sets," Information and Control, vol. 8, no. 3, pp. 338-353, 1965.

[5] Y. Suh, Y. Park, and D. Kang, "Evaluating mobile services using integrated weighting approach and fuzzy VIKOR," PLoS One, vol. 14, no. 6, Article ID e0217786, 2019.

[6] G. Lee, K. S. Jun, and E.-S. Chung, "Group decision-making approach for flood vulnerability identification using the fuzzy VIKOR method," Natural Hazards and Earth System Sciences, vol. 15, no. 4, pp. 863-874, 2015.

[7] Y. Li and Y. Liu, "Extended VIKOR-QUALIFLEX method based on trapezoidal fuzzy two-dimensional linguistic Information for multiple attribute decision-making with unknown attribute weight," Mathematics, vol. 9, no. 1, p. 37, 2021.

[8] T.-H. Chang, "Fuzzy VIKOR method: a case study of the hospital service evaluation in Taiwan," Information Sciences, vol. 271, pp. 196-212, 2014.

[9] T. C. Wang and T. H. Chang, "Fuzzy VIKOR as a resolution for multicriteria group decision-making," in Proceedings of the 11th International Conference on Industrial Engineering and Engineering Management, pp. 352-356, Singapore, 2005.

[10] Z. Wu, J. Ahmad, and J. Xu, "A group decision making framework based on fuzzy VIKOR approach for machine tool selection with linguistic information," Applied Soft Computing, vol. 42, pp. 314-324, 2016. 
[11] K. T. Atanassov, "Intuitionistic fuzzy sets," Fuzzy Sets and Systems, vol. 20, no. 1, pp. 87-96, 1986.

[12] R. Roostaee, M. Izadikhah, F. H. Lotfi, and M. RostamyMalkhalifeh, "A multi-criteria intuitionistic fuzzy group decision making method for supplier selection with VIKOR Method," International Journal of Fuzzy System Applications, vol. 2, no. 1, pp. 1-17, 2012.

[13] P. Gupta, M. K. Mehlawat, and N. Grover, "Intuitionistic fuzzy multi-attribute group decision-making with an application to plant location selection based on a new extended VIKOR method," Information Sciences, vol. 370, pp. 184-203, 2016.

[14] R. Krishankumar, J. Premaladha, K. S. Ravichandran, K. R. Sekar, R. Manikandan, and X. Z. Gao, "A novel extension to VIKOR method under intuitionistic fuzzy context for solving personnel selection problem," Soft Computing, vol. 24, no. 2, pp. 1063-1081, 2020.

[15] R. R. Yager, "Pythagorean membership grades in multicriteria decision making," IEEE Transactions on Fuzzy Systems, vol. 22, no. 4, pp. 958-965, 2013.

[16] M. Gul, M. F. Ak, and A. F. Guneri, "Pythagorean fuzzy VIKOR-based approach for safety risk assessment in mine industry," Journal of Safety Research, vol. 69, pp. 135-153, 2019.

[17] P. Rani, A. R. Mishra, K. R. Pardasani, A. Mardani, H. Liao, and D. Streimikiene, "A novel VIKOR approach based on entropy and divergence measures of Pythagorean fuzzy sets to evaluate renewable energy technologies in India," Journal of Cleaner Production, vol. 238, p. 117936, 2019.

[18] X. Ma, M. Akram, K. Zahid, and J. C. R. Alcantud, "Group decision-making framework using complex Pythagorean fuzzy information," Neural Computing \& Applications, vol. 33, no. 6, pp. 2085-2105, 2021.

[19] B. C. Cuong, Picture Fuzzy Sets-First Results, Neuro-Fuzzy Systems with Applications, Institute of Mathematics, Hanoi, Vietnam, 2013.

[20] P. Meksavang, H. Shi, S.-M. Lin, and H.-C. Liu, “An Extended picture fuzzy VIKOR approach for sustainable supplier management and its application in the beef industry," Symmetry, vol. 11, no. 4, p. 468, 2019.

[21] H. C. Liu and X. Y. You, Green Supplier Evaluation and Selection: Models, Methods and Applications, Springer Nature, Basingstoke, UK, 2021.

[22] F. K. Gündogdu and C. Kahraman, "Spherical fuzzy sets and spherical fuzzy TOPSIS method," Journal of Intelligent and Fuzzy Systems, vol. 36, no. 1, pp. 337-352, 2019.

[23] F. K. Gündogdu and C. Kahraman, "Spherical fuzzy sets and decision making applications," in INFUS 2021: International Conference on Intelligent and Fuzzy Systems, pp. 979-987, Springer, Cham, Switzerland, 2020.

[24] F. K. Gündogdu and C. Kahraman, Decision Making with Spherical Fuzzy Sets: Theory and Applications, Vol. 392, Springer Nature, Basingstoke, UK, 2020.

[25] C. Kahraman, F. K. Gündogdu, S. C. Onar, and B. Oztaysi, "Hospital location selection using spherical fuzzy TOPSIS method," in Proceedings of the 2019 Conference of the International Fuzzy Systems Association and the European Society for Fuzzy Logic and Technology (EUSFLAT2019), Atlantis Press, Prague, Czech Republic, September 2019.

[26] T. Mahmood, K. Ullah, Q. Khan, and N. Jan, "An approach toward decision-making and medical diagnosis problems using the concept of spherical fuzzy sets," Neural Computing \& Applications, vol. 31, no. 11, pp. 7041-7053, 2019.
[27] F. K. Gündogdu and C. Kahraman, “A novel VIKOR method using spherical fuzzy sets and its application to warehouse site selection," Journal of Intelligent and Fuzzy Systems, vol. 37, no. 1, pp. 1197-1211, 2019.

[28] F. K. Gündogdu, C. Kahraman, and A. Karasan, "Spherical fuzzy VIKOR method and its application to waste management," in Proceedings of the International Conference on Intelligent and Fuzzy Systems, pp. 997-1005, Springer, Istanbul, Turkey, July 2019.

[29] D. Ramot, R. Milo, M. Friedman, and A. Kandel, "Complex fuzzy sets," IEEE Transactions on Fuzzy Systems, vol. 10, no. 2, pp. 171-186, 2002.

[30] M. Akram and A. Bashir, "Complex fuzzy ordered weighted quadratic averaging operators," Granular Computing, vol. 6, no. 3, pp. 523-538, 2020.

[31] A. S. Alkouri and A. R. Salleh, "Complex intuitionistic fuzzy sets," AIP Conference Proceedings, vol. 1482, no. 1, pp. 464-470, 2012.

[32] K. Ullah, T. Mahmood, Z. Ali, and N. Jan, "On some distance measures of complex Pythagorean fuzzy sets and their applications in pattern recognition," Complex \& Intelligent Systems, vol. 6, no. 1, pp. 15-27, 2020.

[33] M. Akram, C. Kahraman, and K. Zahid, "Group decisionmaking based on complex spherical fuzzy VIKOR approach," Knowledge-Based Systems, vol. 216, p. 106793, 2021.

[34] M. Akram, M. Shabir, A. N. Al-Kenani, and J. C. R. Alcantud, "Hybrid decision-making frameworks under complex spherical fuzzy N -soft sets," Journal of Mathematics, vol. 2021, Article ID 5563215, 46 pages, 2021.

[35] F. Fatimah, D. Rosadi, R. B. F. Hakim, and J. C. R. Alcantud, "N-soft sets and their decision making algorithms," Soft Computing, vol. 22, no. 12, pp. 3829-3842, 2018.

[36] D. A. Molodtsov, "Soft set theory-first results," Computers and Mathematics with Applications, vol. 37, no. 4-5, pp. 19-31, 1999.

[37] M. Akram, A. Adeel, and J. C. R. Alcantud, "Fuzzy N-soft sets: a novel model with applications," Journal of Intelligent and Fuzzy Systems, vol. 35, no. 4, pp. 4757-4771, 2018.

[38] H. Zhang, D. Jia-Hua, and C. Yan, "Multi-attribute group decision-making methods based on Pythagorean fuzzy $N$-soft sets," IEEE Access, vol. 8, pp. 62298-62309, 2020.

[39] A. Adeel, M. Akram, N. Yaqoob, and W. Chammam, "Detection and severity of tumor cells by graded decision-making methods under fuzzy $N$-soft model," Journal of Intelligent and Fuzzy Systems, vol. 39, no. 1, pp. 1303-1318, 2020.

[40] M. Akram, A. Adeel, and J. C. R. Alcantud, "Hesitant fuzzy Nsoft sets: a new model with applications in decision-making," Journal of Intelligent and Fuzzy Systems, vol. 36, no. 6, pp. 6113-6127, 2019.

[41] M. Akram, A. Adeel, A. N. Al-Kenani, and J. C. R. Alcantud, "Hesitant fuzzy $N$-soft ELECTRE-II model: a new framework for decision-making," Neural Computing \& Applications, vol. 33, no. 13, pp. 7505-7520, 2021.

[42] M. Akram, G. Ali, and J. C. R. Alcantud, "New decisionmaking hybrid model: intuitionistic fuzzy $N$-soft rough sets," Soft Computing, vol. 23, no. 20, pp. 9853-9868, 2019.

[43] M. Akram, A. N. F Al-Kenani, and A. N. Al-Kenan, "A hybrid decision-making approach under complex Pythagorean fuzzy $\mathrm{N}$-soft sets," International Journal of Computational Intelligence Systems, vol. 14, no. 1, pp. 1263-1291, 2021.

[44] M. Akram, M. Shabir, and A. Ashraf, "Complex neutrosophic $N$-soft sets: a new model with applications," Neutrosophic Sets and Systems, vol. 42, pp. 278-301, 2021. 